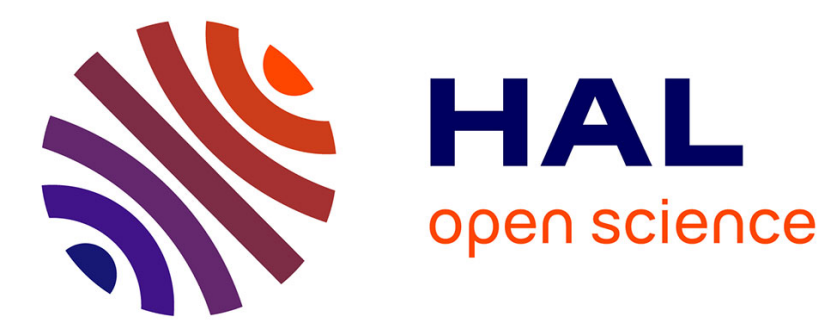

\title{
Striola magica. A functional explanation of otolith geometry
}

Mariella Dimiccoli, Benoît Girard, Alain Berthoz, Daniel Bennequin

\section{To cite this version:}

Mariella Dimiccoli, Benoît Girard, Alain Berthoz, Daniel Bennequin. Striola magica. A functional explanation of otolith geometry. Journal of Computational Neuroscience, 2013, 35 (2), pp.125-154. 10.1007/s10827-013-0444-x . hal-01000762

\section{HAL Id: hal-01000762 https://hal.science/hal-01000762}

Submitted on 4 Jun 2014

HAL is a multi-disciplinary open access archive for the deposit and dissemination of scientific research documents, whether they are published or not. The documents may come from teaching and research institutions in France or abroad, or from public or private research centers.
L'archive ouverte pluridisciplinaire HAL, est destinée au dépôt et à la diffusion de documents scientifiques de niveau recherche, publiés ou non, émanant des établissements d'enseignement et de recherche français ou étrangers, des laboratoires publics ou privés. 


\title{
Striola Magica. A functional explanation of otolith geometry.
}

\author{
Mariella Dimiccoli • Benoît Girard • \\ Alain Berthoz • Daniel Bennequin
}

Received: date / Accepted: date

\begin{abstract}
Otolith end organs of vertebrates sense linear accelerations of the head and gravitation. The hair cells on their epithelia are responsible for transduction. In mammals, the striola, parallel to the line where hair cells reverse their polarization, is a narrow region centered on a curve with curvature and torsion. It has been shown that the striolar region is functionally different from the rest, being involved in a phasic vestibular pathway. We propose a mathematical and computational model that explains the necessity of this amazing geometry for the striola to be able to carry out its function. Our hypothesis, related to the biophysics of the hair cells and to the physiology of their affer-
\end{abstract}

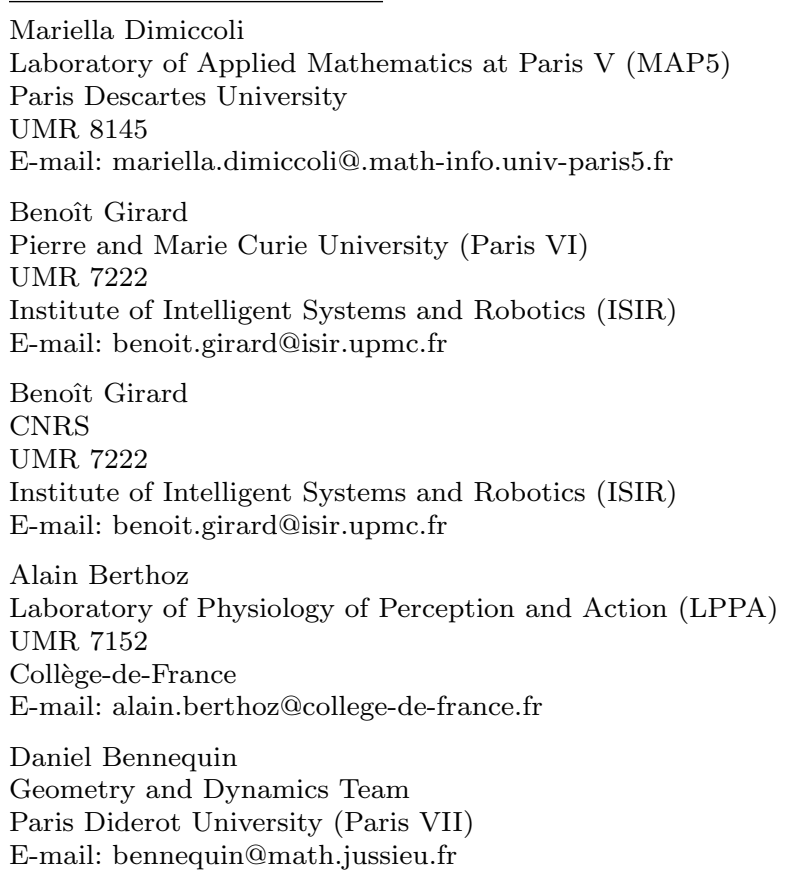


ent neurons, is that striolar afferents collect information from several type I hair cells to detect the jerk in a large domain of acceleration directions. This predicts a mean number of two calyces for afferent neurons, as measured in rodents. The domain of acceleration directions sensed by our striolar model is compatible with the experimental results obtained on monkeys considering all afferents. Therefore, the main result of our study is that phasic and tonic vestibular afferents cover the same geometrical fields, but at different dynamical and frequency domains.

Keywords otolith organs $\cdot$ striola $\cdot$ vestibular pathway

\section{Author summary}

The vestibular end organs in the inner ear have undergone various shape modifications during evolution, depending on the locomotion system and on the ecological niche of each considered species. Specifically, our proposal deals with the geometry of the otolith organs (the saccule and the utricle), and with the twisted band of hair cells known as the striola, running across the otolith epithelial surface (the macula). The present study proposes a functional model of the striola of mammals from which can be proved the necessity for the striola to be centered on a curved and twisted curve. This model supposes that striolar afferents measure the derivative of acceleration directions (jerk), rather than the acceleration directions, by means of the intersection of the receptive fields of several striolar hair cells. It provides an explanation of the observation that in various rodent species the afferents of the striola contact, on average, two hair cells of the striola. Finally, computer simulations of the receptive fields of such a system fit well with experimental data acquired by Fernandez and Goldberg in 1976. We also examined the consequences of our model for the physiology and evolution of the striolar region.

\section{Introduction}

One important problem in Biology is to relate the function of organs with their structure; here we propose to deduce the amazing shape of the hair cell polarization field of vestibular otolith sensors in mammals from simple hypotheses concerning the dynamical function of the hair cells and of their afferent neurons.

Otolith end organs can sense the effect of linear acceleration of the head including gravitation. The structure of these organs has assumed different shapes and organization through the evolution. In mammals, the principal otolith organs are the utricle and the saccule whose epithelial surfaces, named maculae, are approximatively lying on the vertical plane and on the horizontal plane respectively. The receptors on the macula are the hair cells whose 
bundles of stereocilia form oriented arrows, pointing to a longest hair, the kinocilium. Thus each hair cell presents a morphological polarization vector. Under the effect of linear accelerations or gravity, the otolith membranes containing crystals drive a gel and deflect the cilia of the hair cells. The hair cells depolarize when their hair bundles move in the direction of the kinocilium. Then the hair cells vary their potential according to linear accelerations, modulating the firing rate of sensory afferent neurons that transmit information to vestibular nuclei and to the cerebellum. Furthermore, this sensory system is actively modulated by efferent projections on the hair cells or on their afferent synapses.

The population of hair cells is not homogeneous: in particular larger and more isolated cells are distributed along a narrow central zone, named the striola [19], [12], [35]. Most of the striolar hair cells have specific immunohistochemical properties, in particular they express Calmodulin, Calbindin, Calretinin, Parvalbumin (Oncomudulin) [33], [12], [62]. From these properties we see that in mammals hair cell bundles reverse their polarity on a line parallel to the striola, named the polarity reversion line (PRL). The striola has a characteristic shape: on the utricle, it has a $C$ shape similar to a circular or parabolic arc, depending on the species, whereas on the saccule it has an $S$ shape that justifies the frequent comparison with a hook (see Fig. 1).

In addition, it has been observed since long time that the macular surfaces are far from being flat. The surface of the saccule is described as an ellipsoidal lens with its convexity laterally oriented, whereas the surface of the utricle looks like the upside palm of an hand (see [67], [59], [44], [45], [69], [70] for humans and [8] for guinea pig). The curvature of the maculae in the three dimensional space is suspected to be useful for detecting a wide range of linear accelerations [27], [45]. The idea that the geometry of the epithelium, of the hair bundles and the synaptic arrangement with afferent cells, allied to physiology and dynamics, is essential for information processing in the vestibular maculae was developed in particular by Tomko et al. [68] and Ross et al. [51], [55].

Most of the hair cells near the striola are encapsuled in calyces by their sensory afferents, they possess specific ionic channels (in particular a delayed rectifier $g_{K L}$, activated at resting potential) that make them non-linear and difficult to activate. These hair cells are said of type I. Their afferents are phasic, adapting and irregular (i.e. they have a large coefficient of variation of interspike time, 30\%). On the contrary, in extrastriolar regions, most hair cells are of type II: they are linear and easily activated, with afferents that are tonic, less adapting and more regular (mean coefficient of variation 3\%) (see Fig. 2). Complex calyx endings are more numerous on the striola [12]. However type I and type II hair cells are found everywhere on the macula, in different proportion, depending on the species [12], and there is no strict correspondence between type I and striola or type II and periphery. In fact the physiology of both types of hair cells depends on the region where they are located, as explained in Goldberg [21] and Eatock and Songer [14]. Goldberg et al. [22], have shown that the striolar afferent system is more sensitive and 


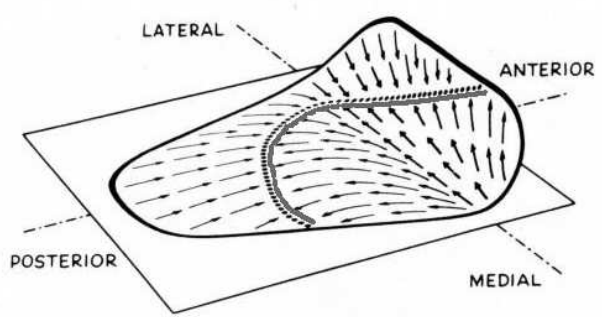

A

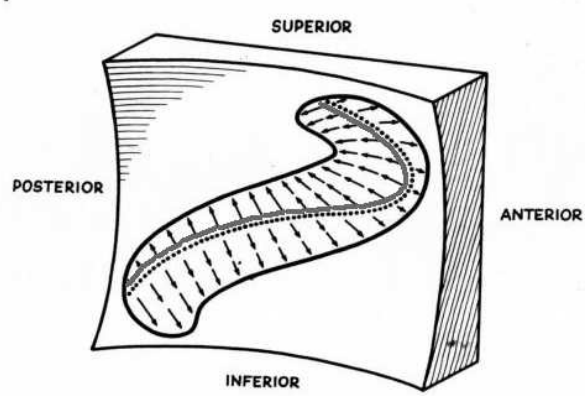

PRL:

Striola:
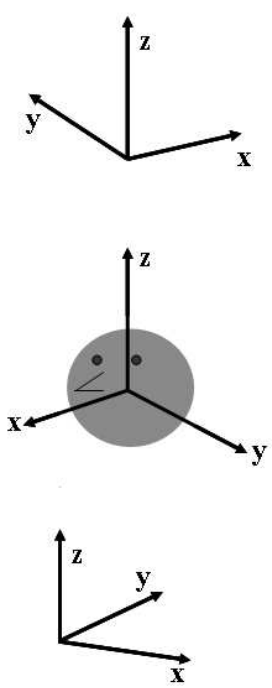

B

Fig. 1 Adapted from Spoedlin, 1966 [64]. Macula of the left utricule $(A)$ and of the right saccule $(B)$ with their morphological polarization vectors. The insets illustrate the orientation of the respective coordinate system: $\mathrm{x} / \mathrm{y} / \mathrm{z}$ indicates front/left/up, respectively. The striola is parallel to the PRL where the morphological polarization vectors invert their polarity. Therefore, all morphological polarization vectors along the striola have the same orientation: they are oriented along the positive $Y$-axis for the utricule and along the positive $Z$-axis for the saccule.

phase advanced, i.e. it detects a signal between the linear jerk and the linear acceleration. They observed that even type II hair cells on the striola have a similar bias. Also the morphology of hair bundles of all the types vary from the striola to the periphery [58], [65]. According to one of the first observers, H.H. Lindeman ([37], [38]): The regional differences in the structure of the maculae suggest that the striola differs functionally from the peripheral areas. But this function is still largely mysterious.

Our aim is to present a functional model of the striola that supports the conjecture of Lindeman [38], in the light of many experimental and theoretical studies ([16], [17], [22], [53], [12], [58], [29], [14]).

Our first hypothesis is that striolar type I hair cells provide a non-linear tuning of acceleration vectors with maximal response when they are perpendicular to the striola curve at their place. Our second hypothesis is that the striolar afferents contacting these hair cells react non-linearly to the infor- 


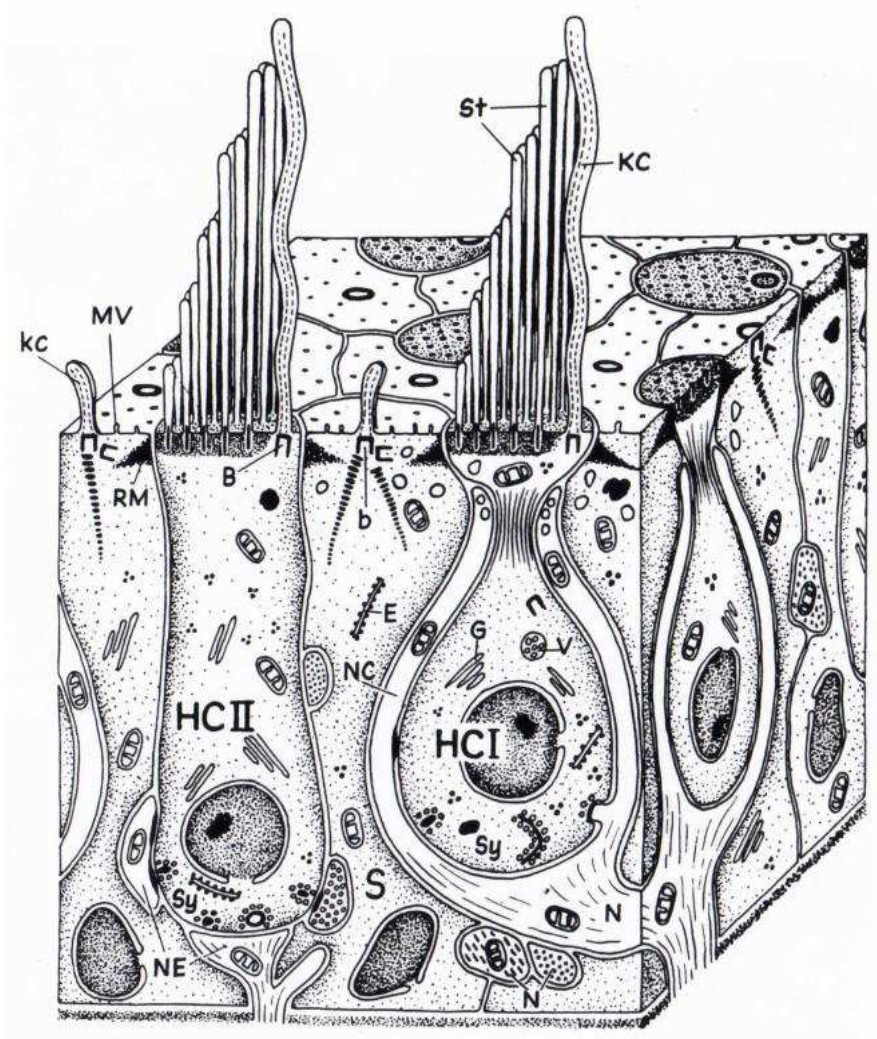

Fig. 2 Reprinted from Spoedlin, 1966 [64]. Vestibular sensory epithelium and its innervation: HCI and HCII correspond to the hair cells of type I and type II respectively; St and $\mathrm{KC}$ indicate the stereocilia and kinocilia respectively; $\mathrm{NC}$ refers to the calyces.

mation given by the intersection of the receptive fields of the hair cells they contact. These afferents can be modulated by striolar type II hair cells, that provide complementary information about the planes containing a given acceleration vector. We neglect this modulation in our computational study.

Moreover, to select the set of pairs of hair cells on the striola which are contacted by afferent neurons, we make the hypothesis that, at the level of population, the afferent neurons should represent an uniform map for the possible variations of acceleration directions.

Henceforth we refer to as variation of a direction, any vector perpendicular to this direction. This corresponds to a vector tangent to the sphere representing all the directions in the space. The first prediction of our model is that 
the striola must be centered on a curve which has curvature and torsion. A second prediction is that the domain of acceleration directions detected by the striolar system for the phasic pathway, and the jerk information, has the same size as the domain detected by the extrastriolar region for the tonic pathway and the acceleration information.

Thus, we suggest that functionally the striola plays the role of a virtual macula, additional to the real macula (see the appendix for the precise definition of this functional surface).

This work has potential applications in motor control and in medicine, since it describes a part of the subsystem of the vestibular system that is probably responsible of the highest order anticipation, and that is also the most fragile during advanced age and under antibiotic treatment [37], [41]. Also specific regeneration of hair cells occurs in the striolar and juxtastriolar regions of the utricle [36].

The structure of the paper is as follows: Section 3 provides a presentation of the mathematical model and gives the equations for the receptive fields model of the hair cells and the afferents. Section 4 provides a detailed description of the simulations performed to validate the proposed model. In particular, it explains how to select a subpopulation for optimal detection of acceleration changes, it shows the statistical characteristics of this population, and it describes the learning algorithm for decoding. Finally, section 4 provides our interpretations of the present work, in relation to phylogenetic data as well as to its biological relevance in view of previous work. An appendix 7 introduces to the differential geometry underlying the model.

\section{Method}

\subsection{A functional model of the striola}

On the striola, the morphological polarization vectors of the hair bundles vary continuously: in particular they are not disposed head to tail as they are on the PRL. This is true for mammals, as shown by Desai et al. [12], and Tribukait et al. [70], and for birds ([61], [74]). Thus, we assume that the polarization of hair cells is well defined and continuous on the striola curve. On the utricle, the PRL has no intersection with the striola [14], with depolarization of the hair cells induced by laterally oriented acceleration vectors. On the saccule, recent observations of Songer on rats ([14]), indicate the existence of two striolae with opposite polarization. However, we will present the results for only one striola on the saccule, with depolarization induced by acceleration vectors directed to the top of the head, which corresponds to a striola located above the PRL.

A priori the arrangement on a narrow band should restrain the detected acceleration directions to a narrow domain. However the detected domain can 
be enlarged if several hair cells on the striola can join their information. It is an experimental observation that many striolar afferent dendrites envelop several hair cells in their calyces. One possibility for joining information is that each afferent cell signals a certain linear combination of different projections of the acceleration vector, thus proceeding by averaging (or by addition). A second possibility is that each afferent estimates an acceleration by intersecting several sectors signaled by its input hair cells, thus proceeding by exclusion (or by product). A more general solution is to use a combination of both models, using complex afferent microcircuits, as observed by Ross et al.[52], [54], but in the present study we consider only not hybrid models.

Both not hybrid fusion models enlarge the detected domain of acceleration directions. By geometrical analysis and numerical simulations we observed that the multiplication model gives a much larger detected domain than the addition model. (see section 4 )

Thus we suggest that a typical afferent compute the intersection of the domains of the hair cells it contacts. Let us explain with elementary formulas the consequence of this rule for the striolar function.

We suppose that the striola is a band centered on a twisted curve $\mathcal{C}$, described in a cartesian coordinates system fixed to the head by the parameterization

$$
x=f(u), \quad y=g(u), \quad z=h(u),
$$

where $u$ is a real parameter, $f, g, h$ are real smooth functions, $x$ goes in front, $y$ laterally and $z$ upside. If the acceleration vector of the head, denoted by $\mathbf{A}$, has coordinates $a, b, c$, the scalar product with the tangent $\mathbf{T}$ of $\mathcal{C}$ in a point $\mathcal{C}(u)$ is given by

$$
\mathbf{A} . \mathbf{T}=f^{\prime}(u) a+g^{\prime}(u) b+h^{\prime}(u) c .
$$

(where a prime denotes a derivative with respect to $u$ ). The maximum activation of one hair cell is attained only when $u$ makes the scalar product $\mathbf{A}$.T equal to zero, which corresponds to:

$$
f^{\prime}(u) a+g^{\prime}(u) b+h^{\prime}(u) c=0,
$$

with additional inequalities telling that the vector $(a, b, c)$ has a positive scalar product with the polarized normal of the striola curve in the macula surface. An afferent cell contacts two hair cells, located at two different values of the parameter $u$, say $u_{1}, u_{2}$, consequently, the preferred acceleration direction of the afferent cell is $(a, b, c)$ if and only if $u_{1}, u_{2}$ are the solutions of the above equation (3).

Since the space of directions has two independent dimensions, the best curves to represent in the two dimensional space the set of directions are the curves that give a pair of solutions $\left(u_{1}, u_{2}\right)$ of $(3)$ for each unit vector $\mathbf{A}$ belonging to the largest possible solid angle.

It is easy to demonstrate (see 7 ) that the curve $\mathcal{C}$ has to be curved and twisted 
for that. The simplest example, that also gives locally all curved and twisted curves, is given by the normal rational curve, also named twisted cubic

$$
x=\alpha u \quad y=\frac{\beta}{2} u^{2}, \quad z=\frac{\gamma}{3} u^{3} .
$$

where $u$ is a real parameter. The corresponding parametrization of direction is given by solving the binomial equation:

$$
\gamma c u^{2}+\beta b u+\alpha a=0
$$

In this case, we get two solutions $u_{1}, u_{2}$ precisely when the discriminant is strictly positive, that is

$$
\beta^{2} b^{2}-4 \alpha \gamma a c>0 .
$$

Thus the method works well for the directions lying outside a convex cone of revolution.

\subsection{Model equations of the striola}

We model the striola of the otolith organs by a parameterized curve $\mathcal{C}: u \mapsto$ $(f(u), g(u), h(u))$, where the parameter $u$ belongs to a closed interval $\left[u_{\min }, u_{\max }\right]$ on the real line, and its image belongs to the three-dimensional space with cartesian coordinates fixed to the head. The $X$-axis points out of the nose, the $Y$-axis out the left ear and the $Z$-axis to the top of the head (see Fig.1).

We assume that the surface representing the macula where the striola lies on is spherical. In fact, considering the extrastriolar system, which detects more static accelerations, the principle of uniform detection predicts a macula surface with the largest possible number of symmetries induced by isometries of the three dimensional space. Since the maximum number of dimensions for a group of symmetries preserving a surface in a three-dimensional space is 3, this corresponds to a piece of sphere or a plane. Since a plane cannot contain a twisted curve, we have taken a spherical lens for the macula. It is worth to remark that the properties described above agrees with many anatomical observations made on vertebrates ([38], [12], [70], [61], [74]).

Taking into account the reported shape of the striola in humans [69],[70], our model for $\mathcal{C}$ was the intersection of a spherical lens with a cylinder on a cubic graph for the saccule, and the intersection of a spherical lens with a cylinder on a circular arc for the utricle. The spheres and cylinders orientations in our simulations correspond to the axis computed by Naganuma et al. [44], [45]. (see Fig. 1).

Let $\mathcal{S}^{2}$ be the two-dimensional sphere of radius $R$ centered at the origin of the frame $O X Y Z$.

In the case of the utricle, the curve representing the striola and lying on $\mathcal{S}^{2}$ is given by the parametric equations:

$$
f(u)=u, g(u)=\sqrt{r^{2}-\left(u-x_{c}\right)^{2}}+y_{c}, h(u)=\sqrt{R^{2}-u^{2}-g(u)^{2}},
$$



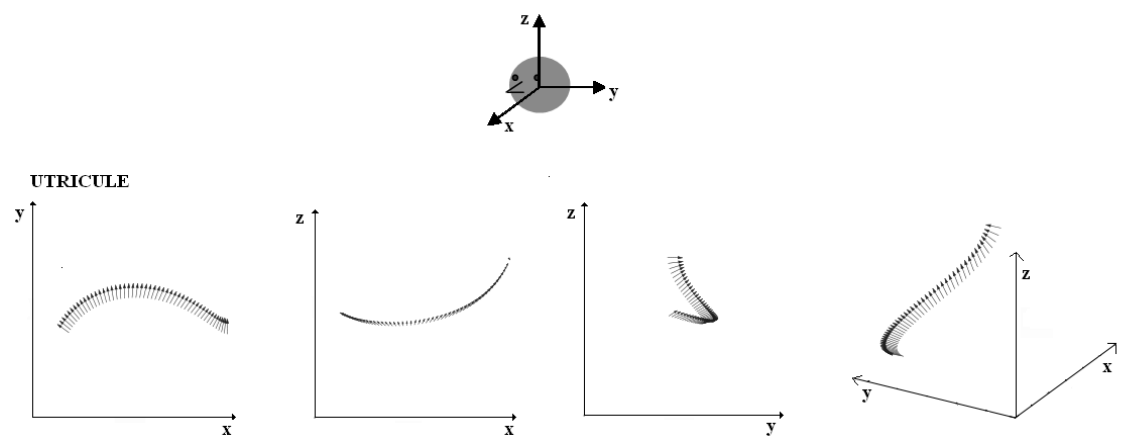

Fig. 3 From left to right are shown the horizontal, sagittal, frontal and three dimensional views of the vectors representing the direction of the kinocilium of the hair cells along the modeled striola of left saccule.
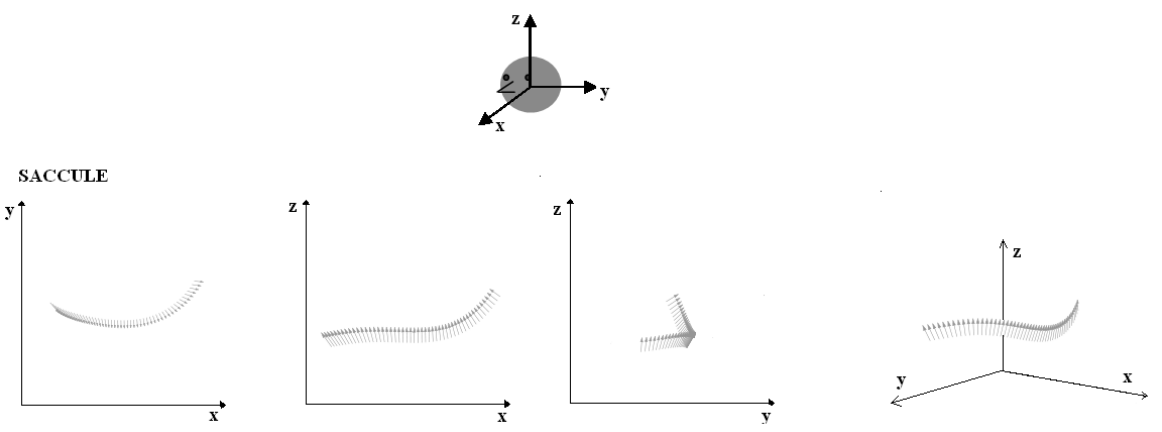

Fig. 4 From left to right are shown the horizontal, sagittal, frontal and three dimensional views of the vectors representing the direction of the kinocilium of the hair cells along the modeled striola of left saccule.

where $g(u)$ represents an arc of circle of center $\left(x_{c}, y_{c}, 0\right)$ and radius $r$.

In the case of the saccule, the curve representing the striola and lying on $\mathcal{S}^{2}$ is given by the parametric equations:

$$
f(u)=u, g(u)=-\sqrt{R^{2}-u^{2}-h(u)^{2}}, h(u)=c u^{3}+\epsilon u,
$$

where $h(u)$ is a cubic polynomial.

The equation for the utricular striola reproduces the known convex shape in the horizontal plane and its known anterior upward inflexion. The cubic equation for the saccular striola corresponds to the known inflexion in the sagittal plane and to its medial curvature. The parameters of these curves have been chosen to be similar to available experimental data about the shape of the left utricular ([67], [69]) and saccular striola ([67], [45], [70]) of humans. For the left utricle we have taken: $\left(x_{c}, y_{c}, 0\right)=(4,0,0), r=5, R=8$ and $u \in[-1.0,6.8]$. In addition the curve has been rotated of -0.3 radians ( -17.19 degrees) with respect the $X$-axis, of -0.4 radians ( -22.92 degrees) with respect the $Y$-axis 
and of -0.5 radians ( -28.65 degrees) with respect the $Z$-axis (see Fig.3). For the left saccule we have taken: $c=0.014, \epsilon=0.01, R=10$ and $u \in[-5,5]$. In addition the curve has been rotated of -0.53 radians ( -30.37 degrees) with respect the $X$-axis and of $\pi$ radians (180 degrees) with respect the $Y$-axis.

In Fig. 3 and Fig. 4 are shown respectively the morphological polarization vectors associated to the striola of the utricle and of the saccule on the left side of the head. They correspond to the arrows in Fig.1, where a three-dimensional view of the macular surfaces of the left utricle (a) and saccule (b) are shown together with their morphological polarization vectors. All morphological polarization vectors on the striola are oriented along the positive $Y$-axis for the utricle and along the positive $Z$-axis for the saccule.

The location of hair cells along the striola is computed by taking the arc length $l(u)$ of the curve $\mathcal{C}$,

$$
s=l(u)=\int_{0}^{u}\left\|\mathcal{C}^{\prime}(v)\right\| d v,
$$

where $\mathcal{C}^{\prime}$ is the velocity vector of the curve $\mathcal{C}$. By using $s$ as new parameter, the curve $\mathcal{C}$ can be written as:

$$
\mathcal{C}: s \mapsto(F(s), G(s), H(s)),
$$

where $s$ varies in $\left[s_{\min }, s_{\max }\right] \subset \mathbb{R}$ and where $F(s)=f\left(l^{-1}(s)\right), G(s)=$ $g\left(l^{-1}(s)\right)$ and $H(s)=h\left(l^{-1}(s)\right)$.

In the following we consider that several hair cells can have the same parameter $s$, thus we model a narrow band of cells and not only a one-dimensional array of cells, but we neglect the effects of the width of the band.

The discretized version of the curve $\mathcal{C}$ consists of a finite set of equidistant points $C=\left\{\mathcal{C}(0), \mathcal{C}\left(\frac{L}{N}\right), \ldots, \mathcal{C}\left(\frac{L(N-1)}{N}\right)\right\}$, where $N=50$ is the number of modeled hair cells and $L$ is the length of the curve $\mathcal{C}$. In the following we denote by $s_{i}$ the parameter $(i-1) \frac{L}{N}$ for $i=1, \ldots, N$.

The curve $\mathcal{C}$ is equipped with a vector field $\mathbf{N}(s)$ normal to the tangent $\mathbf{T}(s)$ of $\mathcal{C}$ and tangent to $\mathcal{S}^{2}$. If we denote by $\mathbf{R}(s)$ the vector normal to $\mathcal{S}^{2}$, the vector field $\mathbf{N}(s)$ is obtained as the normalized vector product: $\mathbf{N}(s)=\frac{\mathbf{T}(s) \times \mathbf{R}(s)}{\|\mathbf{T}(s) \times \mathbf{R}(s)\|}$. The sign of $\mathbf{N}$ depends on the signs chosen for $\mathbf{T}$ and $\mathbf{T}$ and on the orientation $O X Y Z$. We adapted these choices in such a manner that the vector field $\mathbf{N}(s)$ represents the morphological polarization vectors along the striola.

\subsection{Response of single hair cells}

We assume that the type I hair cells along the striola have non-linear receptive fields, which make them more sensitive to acceleration vectors orthogonal to the striola than a cosine tuning would predict. This is compatible with the simulation results of Nam et al. [47], that we will consider in section 5 . 
Denoting by $\mathbf{A}$ the linear acceleration of the head, we call $\alpha_{i}=\alpha\left(s_{i}\right)$ the angle between $\mathbf{A}$ and the vector $\mathbf{T}_{i}$ tangent to the curve $\mathcal{C}$ at the point $s_{i}$, and $\beta_{i}=\beta\left(s_{i}\right)$ the angle between $\mathbf{A}$ and the vector $\mathbf{N}_{i}$ normal to the curve $\mathcal{C}$ and tangent to the surface of the sphere at the point $s_{i} \in \mathcal{C}$.

The response of a single hair cell of parameter $s_{i}$ to the acceleration stimulus $\mathbf{A}$ is given by the product of two functions:

$$
R\left(s_{i}, \mathbf{A}\right)=f_{1}\left(\alpha_{i}\right) f_{2}\left(\beta_{i}\right) .
$$

The function $f_{1}$ expresses the dependency of the instantaneous response of a single hair cell at $\mathcal{C}\left(s_{i}\right)$ with respect to the angle $\alpha_{i}$. We choose $f_{1}\left(\alpha_{i}\right)$ as follows

$$
f_{1}\left(\alpha_{i}\right)=\sin ^{8}\left(\alpha_{i}\right)
$$

This function (see Fig. 5 (a)) is defined in $[-\pi, \pi]$ and assumes maximum value when $\alpha_{i}=\frac{\pi}{2}$ and $\alpha_{i}=-\frac{\pi}{2}$. This means that $f_{1}\left(\alpha_{i}\right)$ is maximum when the acceleration vector lies on the plane normal to the tangent to the striola at $\mathcal{C}\left(s_{i}\right)$. The exponent 8 was chosen to introduce a strong non-linearity in the transversal tuning of the hair cell. The modeling study of Nam et al. [47] reported a non-linear behavior of this kind, with a flat minimum of the response for right angle stimulations. However, our equation does not model the reported symmetric plateau around the angle of maximum response.

The function $f_{2}$ expresses the dependency of the instantaneous response of a single hair cell of parameter $s_{i}$ with respect to the angle $\beta_{i}$. We choose $f_{2}\left(\beta_{i}\right)$ as follows

$$
f_{2}\left(\beta_{i}\right)=\frac{1}{2} \cos \left(\beta_{i}\right)\left(1+\operatorname{erf}\left(3\left(\frac{\pi}{2}-0.4-\beta_{i}\right)\right)\right)
$$

where $\operatorname{erf}$ is the error function

$$
\operatorname{erf}(z)=\frac{2}{\sqrt{\pi}} \int_{0}^{z} e^{-t^{2}} d t
$$

This function (see Fig.5 (b)) is defined in $\left[0, \frac{\pi}{2}\right]$ and assumes maximum value when $\beta_{i}=0$. Therefore, the response is maximum when the acceleration vector has the same orientation of the morphological polarization vector $\mathbf{N}_{i}$. It represents the standard cosine tuning in the polarization direction.

Therefore, in the plane normal to the striola, the response $R\left(s_{i}, \mathbf{A}\right)$ is maximum when the acceleration vector $\mathbf{A}$ is oriented to the kinocilium.

The proposed activation function does not take into account the intensity of the acceleration $\mathbf{A}$ : a complete model should introduce a sigmoid function $\sigma$ with a threshold, for measuring the dependency in the norm $a=\|\mathbf{A}\|$, giving:

$$
\widetilde{R}\left(s_{i}, \mathbf{A}\right)=\sigma\left(a f_{1}\left(\alpha_{i}\right) f_{2}\left(\beta_{i}\right)\right) .
$$

However, in the present study this dependency on the norm and the static non-linearity have little importance, being the acceleration direction the crucial element in the analysis. 


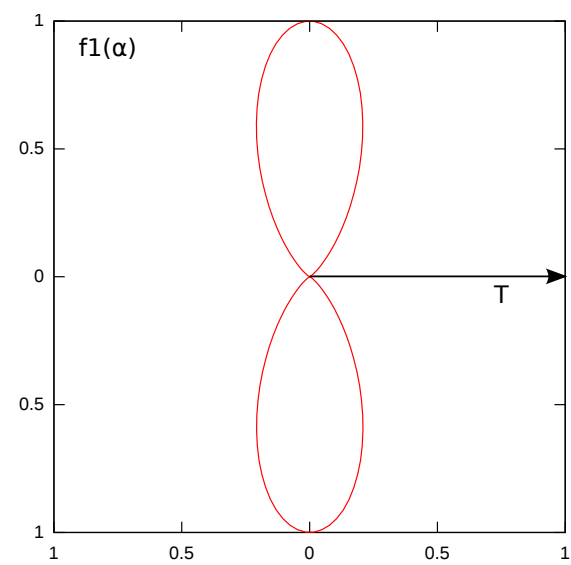

(a)

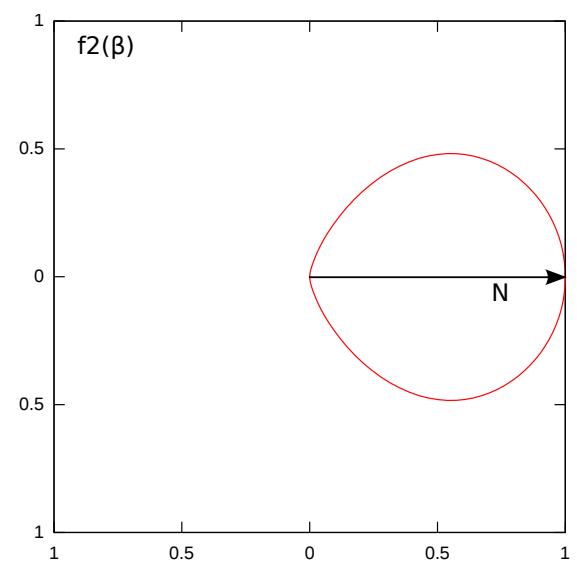

(b)

Fig. 5 (a) Tuning function $f_{1}(\alpha)$ which models the response of a single hair cell $s_{i}$ to an acceleration stimulus, whose direction forms an angle $\alpha$ with with the vector tangent to the striola at $s_{i}$. (b) Tuning function $f_{2}(\beta)$ which models the response of a single hair cell $s_{i}$ to an acceleration stimulus, whose direction forms an angle $\beta$ with the vector normal to striola and tangent to the surface of the macula at $s_{i}$.

\subsection{Response of calyces afferents}

\subsubsection{Single afferent response}

We assume that the striolar afferent neurons integrate non-linearly the activities of two hair cells on average.

The majority of the hair cells in the striolar region of the macula have the particularity of being totally surrounded by a nerve calyx. In our simplified model each afferent neuron takes information from two calyces. We argue that striolar afferents proceed by estimating the acceleration directions as intersection of dihedral sectors, being each sector associated to one of the hair cell captured by a calyx ending of this afferent. Fig. 6 shows how the theoretic preferred direction of the afferent $a_{i j}$ capturing two hair cells of parameters $s_{i}$ and $s_{j}$ is computed geometrically. To each parameter $s_{i}$ is associated a plane determined by its polarization vector $\mathbf{N}_{i}$ and the vector $\mathbf{R}_{i}$ normal to the surface of the macula at $\mathcal{C}\left(s_{i}\right)$. The preferred direction of the afferent $a_{i j}$ is given by the direction of intersection of the planes associated to each hair cell it captures.

For each possible afferent cell $a_{i j}$, the theoretic preferred direction $\mathbf{A}_{i j}=$ $\left(\theta_{i j}, \phi_{i j}\right)$ is given by the vector product $\frac{\mathbf{T}_{i} \times \mathbf{T}_{j}}{\left\|\mathbf{T}_{i} \times \mathbf{T}_{j}\right\|}$.

The response to a given acceleration stimulus $\mathbf{A}$ of a single afferent $a_{i j}$ which takes information from the hair cells in $\mathcal{C}\left(s_{i}\right)$ and $\mathcal{C}\left(s_{j}\right)$ is modeled as 


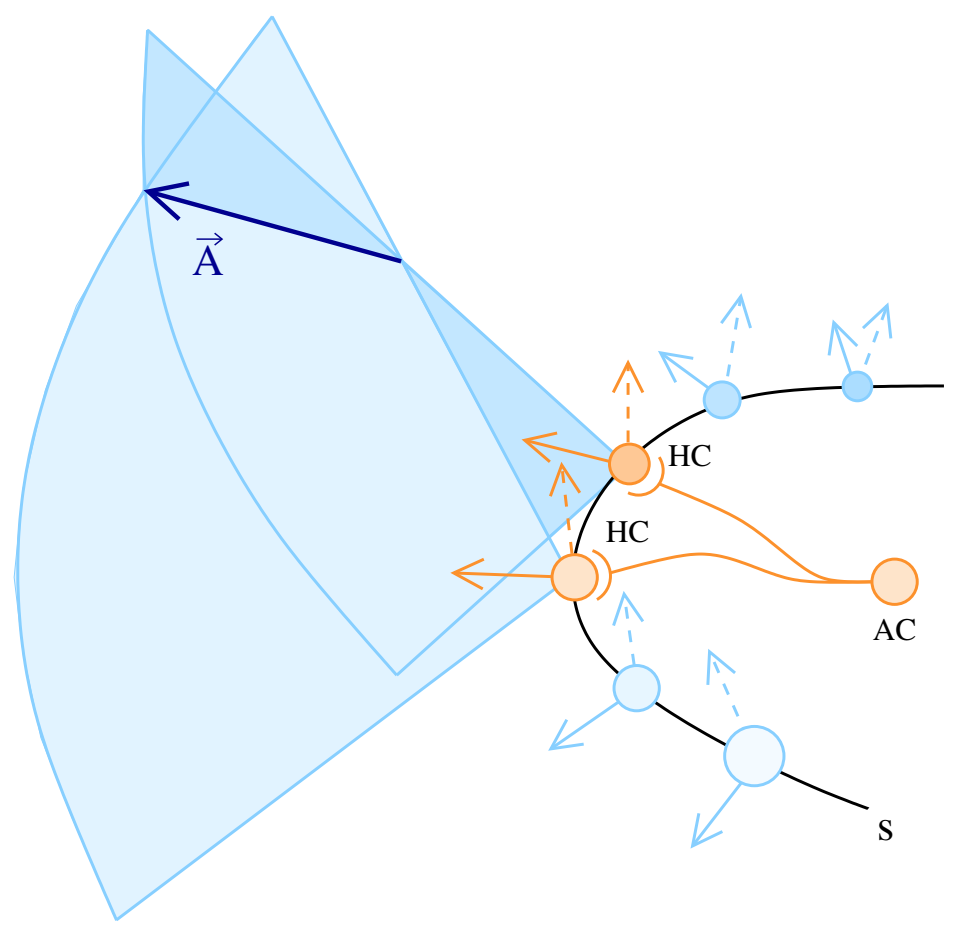

Fig. 6 An afferent cell (AC) encapsulates in calyces two hair cells (HC) on the striola (s). The preferred direction $\mathbf{A}$ of the $\mathrm{AC}$ is given by the intersection of the planes associated to each hair cell, being each plane determined by the polarization vector of the hair cell and by the vector normal to the surface of the macula at the point representing the hair cell.

the product of the responses of the two hair cells:

$$
R\left(a_{i j}, \mathbf{A}\right)=R\left(s_{i}, \mathbf{A}\right) R\left(s_{j}, \mathbf{A}\right)=f_{1}\left(\alpha_{i}\right) f_{2}\left(\beta_{i}\right) f_{1}\left(\alpha_{j}\right) f_{2}\left(\beta_{j}\right)
$$

Therefore, the response of a single afferent is given by the intersection of the set of directions that cause an important excitation of each hair cells.

The dynamic response of the afferent $a_{i j}$ to the acceleration stimulus $\mathbf{A}$ would be described by the following equation:

$$
R\left(a_{i j}, \mathbf{A}\right)(t)=\int f_{1}\left(\alpha_{i}\left(t^{\prime}\right)\right) f_{2}\left(\beta_{i}\left(t^{\prime}\right)\right) f_{1}\left(\alpha_{j}\left(t^{\prime}\right)\right) f_{2}\left(\beta_{j}\left(t^{\prime}\right)\right) \delta_{\epsilon}^{\prime}\left(t-t^{\prime}\right) d t^{\prime},
$$

where $\delta_{\epsilon}^{\prime}\left(t-t^{\prime}\right)=\frac{d}{d t}\left(\frac{1}{\sqrt{2 \epsilon}} e^{-\frac{\left|t-t^{\prime}\right|^{2}}{2 \epsilon}}\right)$, with $\epsilon>0$ is a time wavelet approximating the derivative of the Dirac function. This formula would make the afferent cell able to detect changes of acceleration directions. However, in the following we will only consider the region of acceleration directions and the variations of these directions seen by the afferent cell without testing the response to dynamic stimuli. Roughly speaking, the afferent cell would compute the discrete 
derivative $\frac{\mathbf{A}(t+\delta t)-\mathbf{A}(t)}{\delta t}$ whereas we consider only the response to $\mathbf{A}(t)$ and $\mathbf{A}(t+\delta t)$ separately and we check if the difference between the two responses is large (see section 4.2.3).

In section 5 we present justifications for this kernel, from the known physiology of striolar afferent neurons [14], and from analogy with the global response of semi-circular canals afferent neurons [26].

\subsubsection{Selection of the population of afferent neurons}

From the above striola model equations, we have determined a population of afferents able to detect accurately the variations of linear acceleration. The details of the population selection are given in the section 4, but we expose now the principle underlying our method. We started with all possible pairs of hair cells along the striola: this gave a population of afferent $\mathcal{P}$. Then we computed the domain $\Omega$ of acceleration direction which are detected above a threshold (half of the maximum response), and we selected a sub-population $\mathcal{P}_{\text {resp }}$ of $\mathcal{P}$, detecting acceleration in $\Omega$ above the same threshold. To proceed further we considered the variations of responses when the acceleration stimuli vary, for sensing the linear jerk. We selected a new domain $\Omega^{\prime}$ of acceleration vectors $\mathbf{A}_{k}$ such that the gradient of some afferent response was sufficiently high in one of six directions $\mathbf{V}_{k}^{d}$ orthogonal to $\mathbf{A}_{k}$. Then we defined the subpopulation $\mathcal{P}_{\text {grad }}$ of $\mathcal{P}_{\text {resp }}$ which can detect accurately the variations of acceleration vectors in sufficiently many directions. We limited the population $\mathcal{P}_{\text {grad }}$ by a uniformity condition, requiring that the number of afferents sensing a given variation $\left(\mathbf{A}_{k}, \mathbf{V}_{k}^{d}\right)$ do not departs too far from the mean number.

\subsection{Decoding}

Based on the response of a population of afferent cells to a given acceleration direction $\mathbf{A}$, the brain should be able to extract an estimate $\hat{\mathbf{A}}$ of the underlying encoded original stimulus $\mathbf{A}$. To verify that the information encoded by our model can be appropriately decoded by the brain, we have used a supervised learning method.

A simple decoding method without learning such as population vector decoding proposed by Georgopoulos [20] would be inappropriate in our case, because this decoding method works well when the patterns of activity as a function of the stimulus behaves like gaussian functions. But in our model the patterns of activity as a function of the stimulus parameter do not follow gaussian-like laws (see 4.2).

A learning algorithm was therefore necessary to discover a regular mapping between the population responses and the underlying stimulus. We have used a supervised learning algorithm, the classical backpropagation algorithm with a 2-layer perceptron [2], in order to map the simulated afferent inputs to the direction outputs. Because this algorithm does not use local learning rules, its 
biological plausibility remains uncertain, and in fact it has been used here as a simple way to assess the decodability, not as a model of any brain operation.

\section{Results}

4.1 The shape of the striola and the number of calyces

First, our model provides an explanation of the observed shape of the striola, namely a 3D curve with curvature and torsion. This is discussed in details in the mathematical appendix, but we can explain this result without using mathematical symbols. Suppose that an afferent fiber branches and contacts two hair cells along the striola curve $S$. This will allow an improvement of the information from these two cells as soon as the sectors seen by the hair cells intersect transversally. This implies a large curvature of $S$. Moreover, in order to get a large solid angle for the possible directions of intersection, the sectors seen by the hair cells have to twist in space, implying a large torsion of $S$.

Due to the known global orientation in space of the maculae ([9], [67], [69], [67], [45], [70]), we obtain a $S$ curve for saccule which has approximately the same disposition in space than the $S$ curve of the utricle of the opposite hemisphere of the brain. Thus, on each side of the brain we have two twisted space curves, and by the union of all these four curves we obtain a curve on a sphere that resembles the division on a tennis ball (or suture of base ball) (see Fig. 7).

A main point in our model is that the striola afferent system forms a map of directions in space by coupling several points along the striola curve. This correspond to the mathematical concept of divisor of a curve, due to Abel and Riemann (see the book of Griffith and Harris [24]). Since directions in the three dimensional space depend on two parameters and points on a curve depend upon only one real parameter, in average we must take into account two points on the curve for each direction in 3D space. This agrees with experimental observations: the results of Goldberg et al. [22] give 2.26 as a mean number of calyces by afferent in the utricle of the chinchilla, and the results of Desai et al. [12] give 1.84. These last authors also computed the mean number of calyx terminations of afferents for the saccule and utricle of six species of rodents (mouse, rat, gerbil, guinea pig, chinchilla and tree squirrel): except for mouse and gerbil (around 1.55 and 1.65 respectively) they found indexes larger than 1.75 .

Thus our model gives an explanation of the observed mean number of calyces for striolar afferent fiber.

As a consequence we conclude that the striolar system can detect three dimensional acceleration directions and their change in time (jerk) without the need of computing the intensity of the sensed accelerations. 


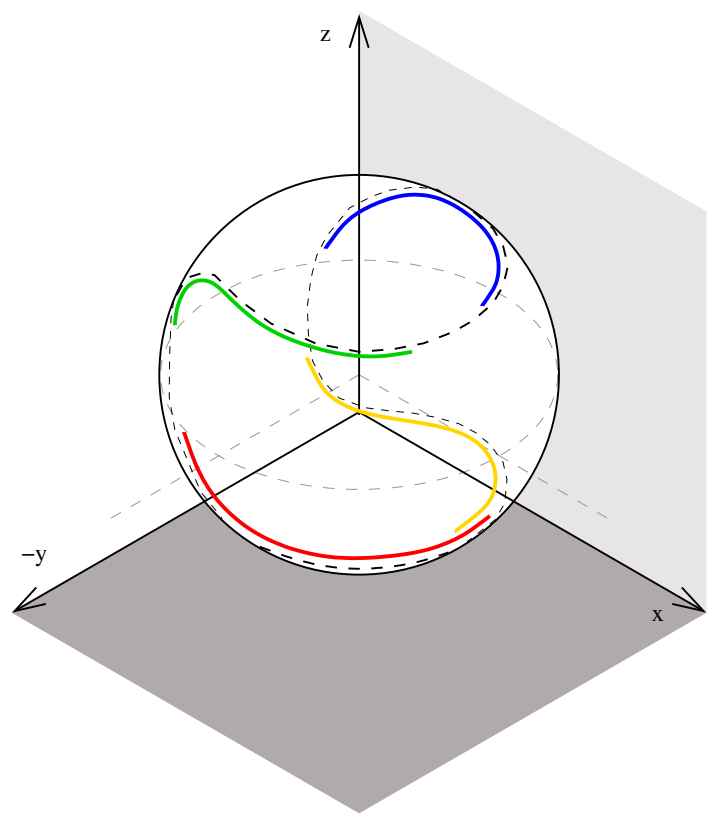

Fig. 7 The red and the yellow curves represent respectively the striola of the right and of the left utricule. The green and the blue curves represent respectively the striola of left and of the right saccule.

More refined theoretical results (see 7) allow an improvement of the optimal curve for the striola:

1) If a curve $S$ allows a smooth parametrization in an open solid angle by pairs of points in the vicinity of a point $P_{0}$, then there exists an Euclidian affine change of coordinates in the three dimensional space such that $S$ has a contact of order 4 with a twisted cubic.

2) Let us choose as coordinates for the pairs of points on the curve $S$ the elementary symmetric functions of the curvilinear abscissas, and as measure on the set of directions the Euclidian solid angle. Denoting by $\varphi$ the transformation sending the pairs of points in $S$ to the corresponding directions in the three dimensional space, then, at the second order of approximation in the distance of the points, the jacobian determinant of $\varphi$ is equal to $-\kappa \tau^{2} / 4$, where $\kappa, \tau$ denote respectively the curvature and torsion of the curve. Thus, for curves $S$ in the three dimensional Euclidian space, to obtain the largest uniformity of representation for information maximization, the curve $S$ must have a curvature and a torsion such that the function $\tau \sqrt{\kappa}$ is constant; 3) For curves on a sphere, the optimal curves are the unique spherical curves with given constant product $\tau \sqrt{\kappa}$. They are associated to lemniscatic elliptic functions [23]. 
However, our model does not implement the theoretically optimal striola, but a standard spherical curve with parameters deduced from empirical data.

4.2 The receptive domains

\subsubsection{Multiplication versus addition}

Numerical simulations allowed to compare different rules of cooperation between hair cells. We compared the averaging rule with the intersection rule and we found that the second gives a much larger detected domain than the first, as can be seen on the Fig. 8 .

In Fig. 9 is shown an example of receptive field (response in function of acceleration direction) of an afferent capturing two hair cells. On the first row of Fig. 9, corresponding to the utricle, are represented the responses of two different hair cells ((a) and (b)) and responses of the afferent capturing these two hair cells (Fig. 9 (c)). The same representation holds for the second row, which corresponds to the saccule.

\subsubsection{Responding afferent population}

Another result, obtained through numerical simulations, is the domain of acceleration directions sensd by the striolar system. This has been achieved by assuming the simple forms of maculae and striolae discussed above and by selecting sub-populations of afferent neurons in order to have an uniform detection of the variations of acceleration directions (see Fig. 11).

Let $\mathcal{S}_{A}^{2}$ be the sphere of radius 1 representing all possible acceleration directions. In Fig.11 (a) and (b), we have associated to each $\mathbf{A}_{k} \in \mathcal{S}_{A}^{2}$ the maximum value of the response obtained among all possible afferents. As expected considering the orientation of the morphological polarization vectors along the striola (see Fig.1), we have found functional polarization vectors only on the upper hemisphere for the saccule and only on the left part of both upper and low hemisphere for the utricle (see Fig.11).

We denote by $\Omega$ the region of $\mathcal{S}_{A}^{2}$ for which the global response is above a fixed threshold $\lambda_{R}$ :

$$
\Omega=\left\{\mathbf{A}_{k} \in \mathcal{S}_{A}^{2} \mid \exists a_{i j}: R\left(a_{i j}, \mathbf{A}_{k}\right)>\lambda_{R}\right\}
$$

We simply took for $\lambda_{R}$ the half of the maximum absolute value of the response obtained among all possible afferents for all possible acceleration directions. We 

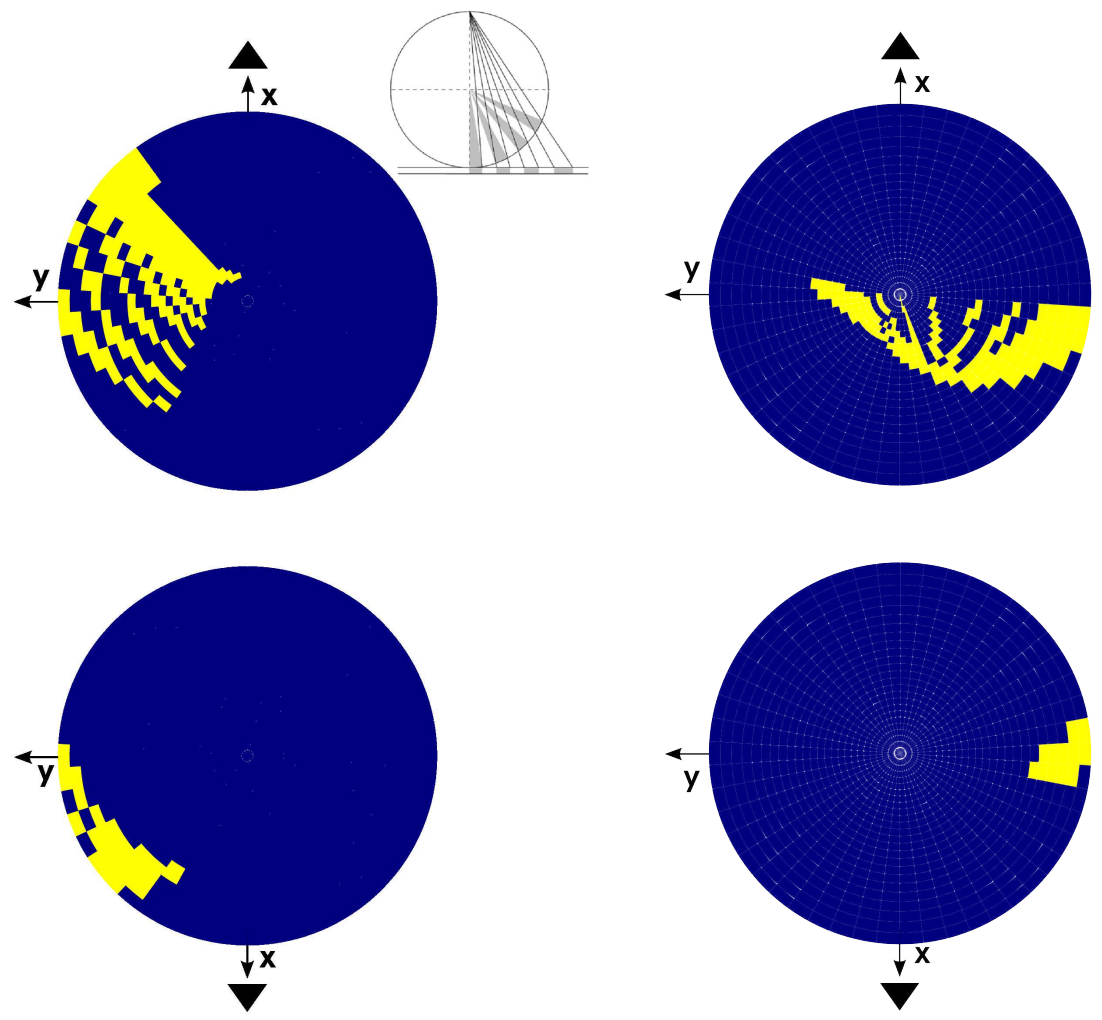

(a)

(b)
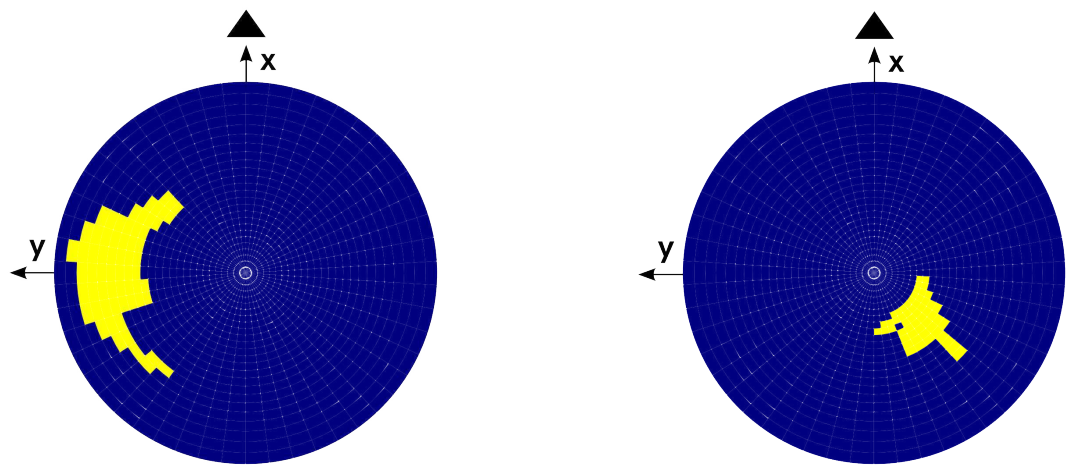

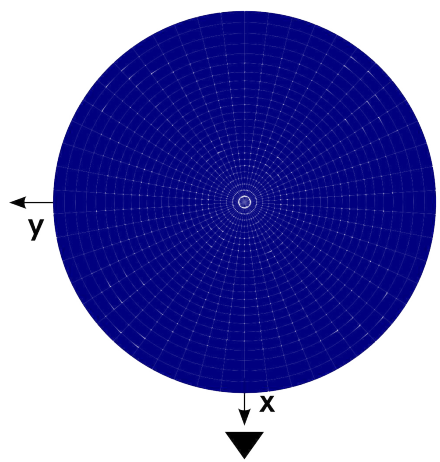

(c)

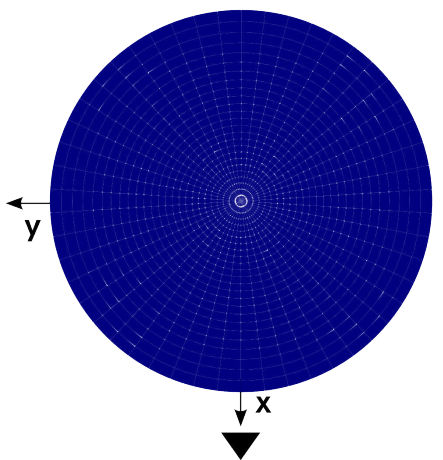

(d)

Fig. 8 The left column represents the domain of acceleration directions computed by all possible pairs of hair cells by intersection of dihedral planes for the utricle (a) and the saccule (c) respectively. The right column represents the domain which would have been detected by taking the vector sum of hair cells activity by pairs (b) and (d) for the utricle (b) and the saccule (d) respectively. The apparent checkerboard pattern is due to the discretization of the population. The inset shows how the north (south) hemisphere is represented with a stereographic projection from the south (north) pole on the plane tangent to the north (south) pole. 


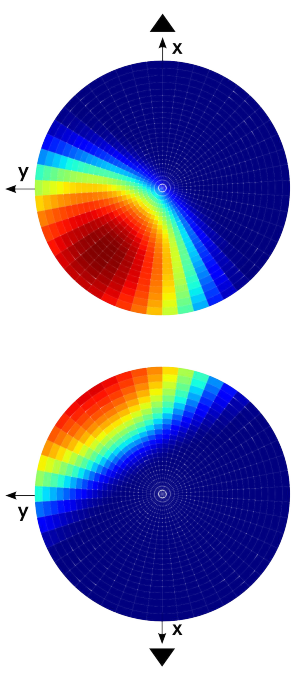

(a)
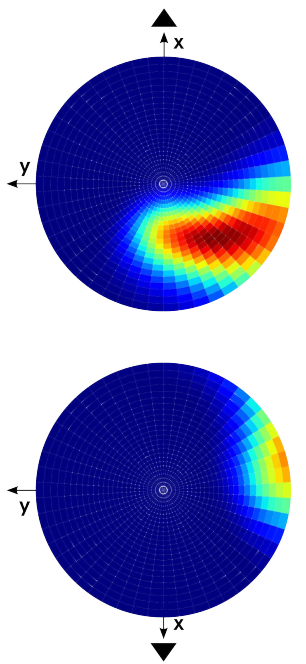

(d)
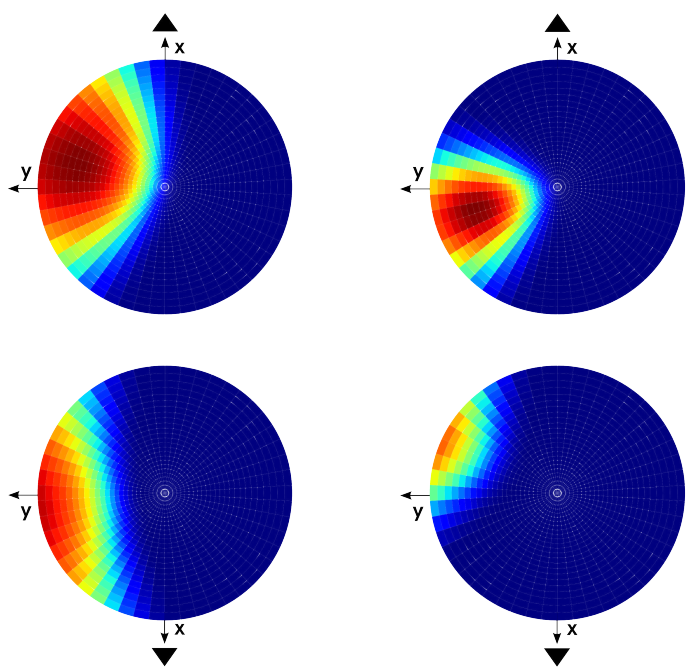

(b)
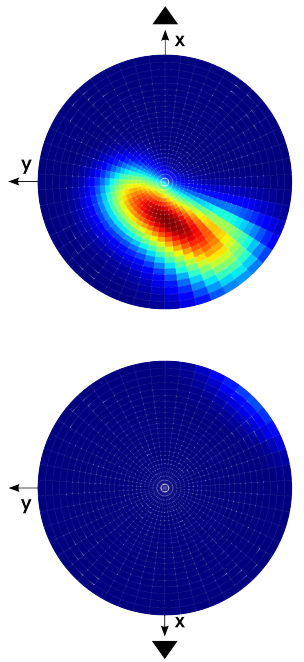

(e)

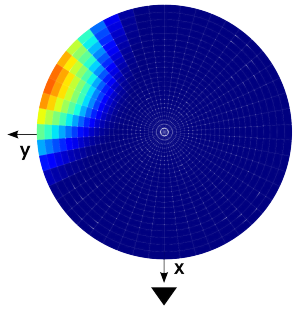

(c)
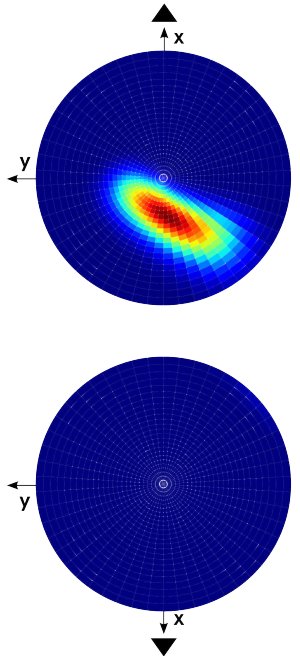

(f)

Fig. 9 On the sphere of all acceleration directions, (a) and (d) represent the receptive field of a single hair cell $s_{i}$ for the utricle and the saccule respectively; in the same manner (b) and (e) represent the receptive field of another single hair cell $s_{j}$ for the utricle and the saccule respectively, then (c) and (f) represent the receptive field of the afferent $a_{i j}$ capturing the hair cells $s_{i}$ and $s_{j}$. 


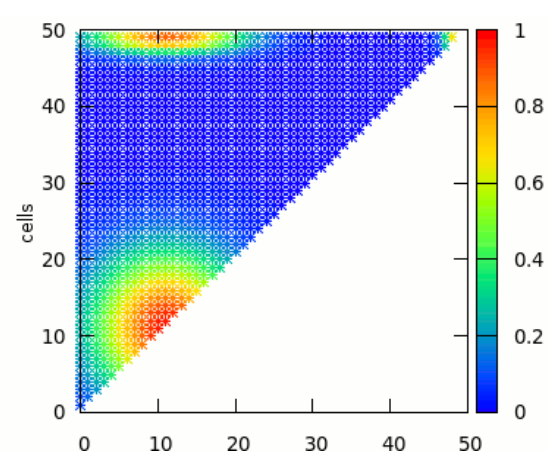

(a)

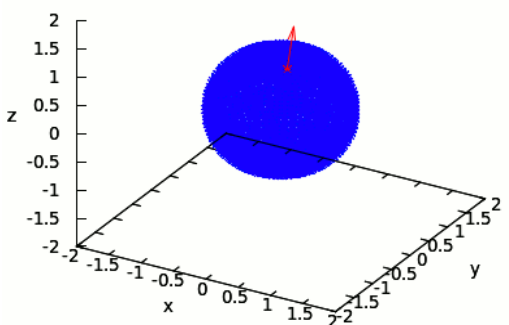

(c)

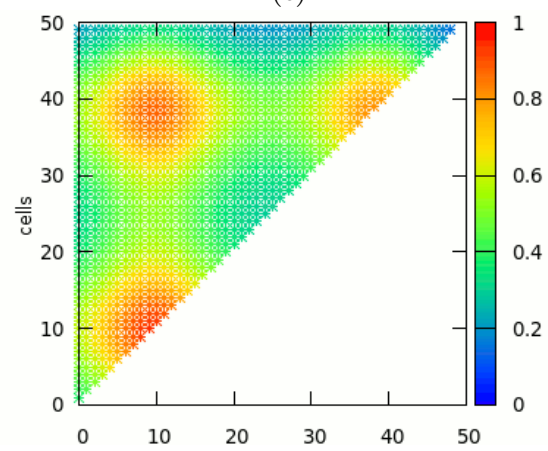

(e)

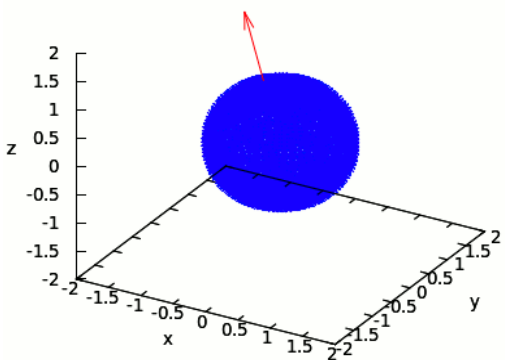

(g)

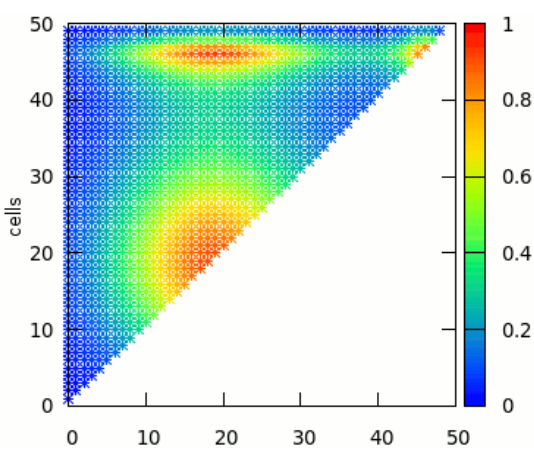

(b)

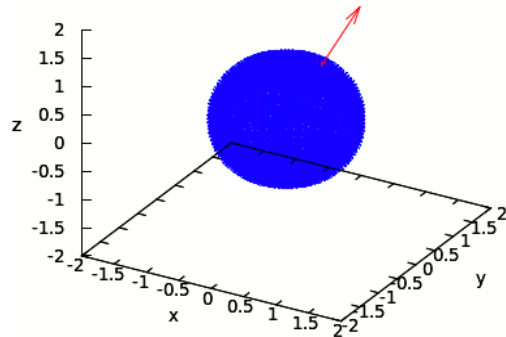

(d)

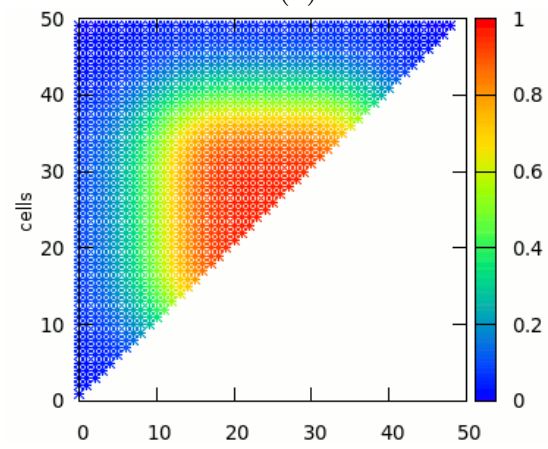

(f)

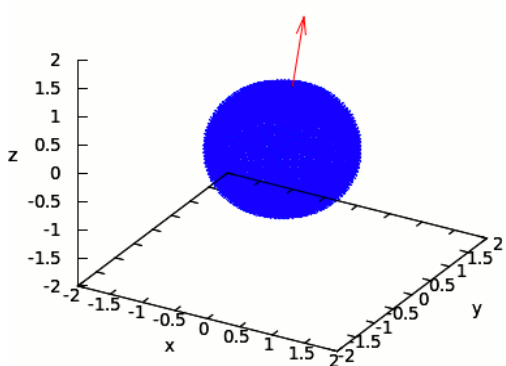

(h)

Fig. 10 (a), (b) for the utricle and (e),(f) for the saccule represent the activity of all possible afferents in response to the acceleration stimulus represented respectively in (c), (d) and (g), (h). In (a),(b),(e), and (f) the abscissa and the ordinate represent the 50 cells modeled on the striola and the color code the activity of the target afferent cell getting input from the respective pair of hair cells. 

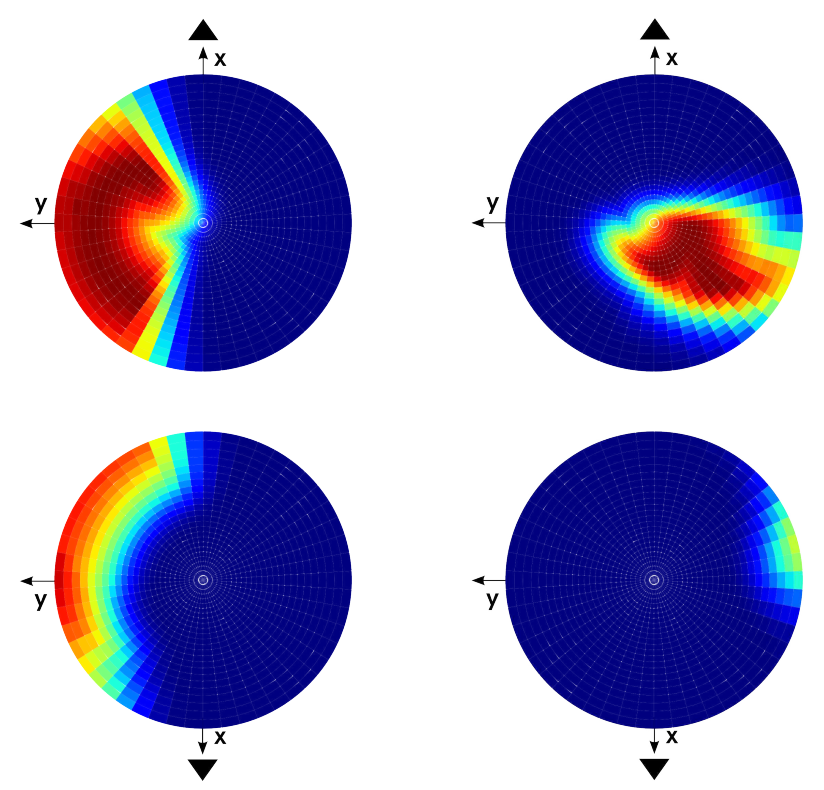

Fig. 11 Maximum value of the response over all possible afferents, normalized with respect to the maximum absolute value, represented on the sphere of all acceleration directions, seen from above and from below, for the utricule on the left column and for the saccule on the right column.

denoted by $\mathcal{P}_{\text {resp }}$ the set of afferents that respond to at least one acceleration in $\Omega$ with an activity above $\lambda_{R}$.

$$
\mathcal{P}_{\text {resp }}=\left\{a_{i j}, i \neq j, \mid \exists \mathbf{A}_{k} \in \Omega: R\left(a_{i j}, \mathbf{A}_{k}\right)>\lambda_{R}\right\}
$$

This had the effect of reducing the dispersion of responses without reducing too much the size of the population.

\subsubsection{Uniform capture of linear jerk}

The acceleration vectors in $\Omega$ are sensed with an intensity range that goes from $\lambda_{R}$ to the maximum absolute value. As it can be observed in Fig.11 ((a) and (b)), the capture is quite uniform in this region. However, since our hypothesis is that the afferents along the striola are best suited to capture the variations of acceleration directions (to sense the jerk), what really matters is that the capture be uniform with respect to a variation of the acceleration directions represented in $\Omega$. Let us describe how we have selected a subset of $\Omega$ and a sub-population of $\mathcal{P}_{\text {resp }}$ to this goal:

First we computed how well a given afferent $a_{i j}$ in $\mathcal{P}_{\text {resp }}$ is able to detect the variation of a given acceleration direction $\mathbf{A}_{k}$ in a given orthogonal direction 
$\mathbf{V}_{k}$ :

For each acceleration $\mathbf{A}_{k} \in \Omega$, we have considered a set of six gradient reference directions $\mathbf{V}_{\mathbf{A}_{k}}^{d}, d \in\{1, . ., 6\}$, randomly chosen and forming a regular hexagon. Note that for each acceleration $\mathbf{A}_{k}$ the set of $\mathbf{V}_{\mathbf{A}_{k}}^{d}$ lies on the plane perpendicular to $\mathbf{A}_{k}$. We associated to each acceleration $\mathbf{A}_{k} \in \Omega$ and to each afferent $a_{i j} \in \mathcal{P}_{\text {resp }}$ the unitary vector gradient $\frac{\nabla_{\mathbf{A}_{k}} R\left(a_{i j}, \mathbf{A}\right)}{\left\|\nabla_{\mathbf{A}_{k}} R\left(a_{i j}, \mathbf{A}\right)\right\|}$, where $\left\|\nabla_{\mathbf{A}} R\left(a_{i j}, \mathbf{A}_{k}\right)\right\|$ is the norm of the gradient of $R\left(a_{i j}, \mathbf{A}_{k}\right)$ with respect to the acceleration direction $\mathbf{A}_{k}$.

$$
\begin{aligned}
\left\|\nabla_{\mathbf{A}} R\left(a_{i j}, \mathbf{A}_{k}(\theta, \phi)\right)\right\| \\
\quad=\sqrt{\left(\frac{\partial R\left(a_{i j}, \mathbf{A}(\theta, \phi)\right)}{\partial \theta}\right)^{2}+\left(\frac{\partial R\left(a_{i j}, \mathbf{A}(\theta, \phi)\right)}{\partial \phi}\right)^{2} \frac{1}{\cos ^{2}(\theta)}},
\end{aligned}
$$

where we take the discretization:

$$
\frac{\partial R\left(a_{i j}, \mathbf{A}(\theta, \phi)\right)}{\partial \theta}=\frac{R\left(a_{i j}, \mathbf{A}_{k_{\theta}}\left(\theta+\delta_{\theta}, \phi\right)\right)-R\left(a_{i j}, \mathbf{A}_{k}(\theta, \phi)\right)}{\delta_{\theta}}
$$

and

$$
\frac{\partial R\left(a_{i j}, \mathbf{A}(\theta, \phi)\right)}{\partial \phi}=\frac{R\left(a_{i j}, \mathbf{A}_{k_{\phi}}\left(\theta, \phi+\delta_{\phi}\right)\right)-R\left(a_{i j}, \mathbf{A}_{k}(\theta, \phi)\right)}{\delta_{\phi}}
$$

We defined $\nu$ as the maximum among all possible afferents in $\mathcal{P}_{\text {resp }}$ of the minimum value of the gradient with respect to an acceleration variation among all accelerations in $\Omega$ :

$$
\nu=\max _{a_{i j} \in \mathcal{P}_{\text {resp }}} \min _{\mathbf{A}_{k} \in \Omega}\left\|\nabla_{\mathbf{A}} R\left(a_{i j}, \mathbf{A}_{k}\right)\right\|
$$

For each gradient response $\nabla_{\mathbf{A}_{k}}\left(R\left(a_{i j}, \mathbf{A}_{k}\right)\right)$ whose norm is above $\nu$, we measured its proximity to the gradient reference direction $\mathbf{V}_{\mathbf{A}_{k}}^{d}$ by computing the angle $\omega=\arccos \left(\nabla_{\mathbf{A}_{k}}\left(R\left(a_{i j}, \mathbf{A}_{k}\right)\right) \cdot \mathbf{V}_{\left.\mathbf{A}_{k}\right)}^{d}\right)$.

We determined that a variation of $\mathbf{A}_{k}$ in the direction $\mathbf{V}_{\mathbf{A}_{k}}^{d}$ is well detected by $a_{i j}$ if $\nabla_{\mathbf{A}_{k}}\left(R\left(a_{i j}, \mathbf{A}_{k}\right)\right) \geq \nu$ and $\omega<\frac{\pi}{6}$. These criteria were retained to insure a sufficiently dense detection of the variations of acceleration directions.

The acceleration vectors appearing for at least one pair $a_{i j}, \mathbf{V}$ form a solid angle $\Omega^{\prime}$ inside $\Omega$, and the afferent appearing for at least one pair $\mathbf{A}, \mathbf{V}$ form a subset $\mathcal{P}^{\prime}$ of $\mathcal{P}_{\text {resp }}$.

If the gradient direction $\mathbf{V}_{\mathbf{A}_{k}}^{d}$ is well detected by an afferent cell the gradient direction $\mathbf{V}_{\mathbf{A}_{k}}^{d+3}$ will also be well detected, because its scalar product with the gradient has the same absolute value and the opposite sign. Therefore the six gradient directions correspond to three independent gradient orientations. The choice of considering only three independent gradient orientation instead of four or more is due to the limited number of hair cells (50), and therefore possible calyx afferents, modeled in the simulations. Nevertheless, we have verified that even when considering six gradient orientations instead of just three 
(that is twelve directions), the afferent cells are well distributed along all these six orientations.

For each afferent $a_{i j}$ we computed the number $N_{i j}$ of times that $a_{i j}$ appears as good detector of a variation of acceleration in $\Omega^{\prime}$. We defined a threshold $\lambda_{N}$ as half of the maximum of the numbers $N_{i j}$ over all pairs $i, j$. The role of this threshold is to prepare a population of afferents with uniform ability to detect the linear jerk. Then, for each $\mathbf{A}_{k} \in \Omega^{\prime}$ we defined the set $\mathcal{P}_{\mathbf{A}_{k}}^{\prime}$ by asking that $a_{i j}$ belongs to $\mathcal{P}^{\prime}$ and that the corresponding $N_{i j}$ is larger than $\lambda_{N}$. And we defined $\mathcal{P}_{\text {grad }}$ as the union of the sets $\mathcal{P}_{\mathbf{A}_{k}}$ over $\mathbf{A}_{k} \in \Omega$.

The final step consists in verifying that all the subsets $\mathcal{P}_{\mathbf{A}_{k}}$ contain almost the same number of elements in $\mathcal{P}_{\text {grad }}$. This corresponds to our uniformity condition.

More precisely, we consider the set of elementary events given by the pairs $\left(\mathbf{A}_{k}, \mathbf{V}_{k}^{d}\right)$ where $\mathbf{A}_{k}$ belongs to $\Omega^{\prime}$ and $d$ varies from 1 to 6 , and we define the random variable $N$ as the number $N_{\mathbf{A}_{k}, \mathbf{V}_{k}^{d}}$ of afferents $a_{i j}$ in $\mathcal{P}_{\text {grad }}$ that are good detectors of the pair $\left(\mathbf{A}_{k}, \mathbf{V}_{k}^{d}\right)$. Then we define $\mu_{\mathbf{A}_{k}}$ as the mean over the variations,

$$
\mu_{\mathbf{A}_{k}}=\frac{1}{6} \sum_{c=1}^{6} N_{\mathbf{A}_{k}, \mathbf{V}_{k}^{d}}
$$

the mean $\mu$ of $N$ is given by

$$
\mu=\frac{1}{M} \sum_{k=1}^{M} \mu_{\mathbf{A}_{k}}
$$

and the standard deviation $\sigma$ of $N$ is given by

$$
\sigma=\sqrt{\frac{1}{M} \sum_{k=1}^{M}\left(\mu_{\mathbf{A}_{k}}-\mu\right)^{2}}
$$

The inequality of Cantelli (also known as the one-sided inequality of Chebyshev), guarantees that in almost every data sample, no more than $\frac{1}{1+r^{2}}$ of the data values can be more than $r$ standard deviations away from the mean. In formulas, if $\mu$ is the expected value of the random variable $N$ and if $\sigma^{2}$ denotes its variance, than for any real number $r>0$, the inequality of Cantelli is

$$
\operatorname{Pr}(\mu-N \geq r \sigma) \leq \frac{1}{1+r^{2}}
$$

We found that, for the population $\mathcal{P}_{\text {grad }}$, the numbers $N_{\mathbf{A}_{k}, \mathbf{V}_{k}^{d}}$ satisfy the inequality $\mu-N_{\mathbf{A}_{k}, \mathbf{V}_{k}^{d}} \geq r \sigma$ for a value of $r$ equals to $r=3$ in the case of the utricle and equals to $r=1$ in the case of the saccule. We computed $\mu-r \sigma=38$ for $r=3$ for the utricle and $\mu-r \sigma=23$ for $r=1$ for the saccule.

The tables on Fig. 4.2.3 show the statistics of $N$ computed on the population $\mathcal{P}_{\text {grad }}$ for the utricle and for the saccule. 


\begin{tabular}{|l|c|}
\hline \multicolumn{2}{|c|}{ UTRICULE } \\
\hline $\min \mu_{\mathbf{A}_{k}}$ & 58 \\
$\max \mu_{\mathbf{A}_{k}}$ & 141 \\
\hline $\min \sigma_{\mathbf{A}_{k}}^{2}$ & 0.000000 \\
$\max \sigma_{\mathbf{A}_{k}}^{2}$ & 0.333333 \\
\hline $\min \mu_{\mathbf{A}_{k}}^{\text {norm }}$ & 356.256717 \\
$\max \mu_{\mathbf{A}_{k}^{n o r m}}^{\text {or }}$ & 1752.198653 \\
\hline $\min \left(\sigma^{2}\right)_{\mathbf{A}_{k} \text { norm }}$ & 0.000001 \\
$\max \left(\sigma^{2}\right)_{\mathbf{A}_{k}}^{\text {norm }}$ & 0.180787 \\
\hline$\mu$ & 96.510689 \\
$\sigma$ & 21.614370 \\
\hline
\end{tabular}

\begin{tabular}{|l|c|}
\hline \multicolumn{2}{|c|}{ SACCULE } \\
\hline $\min \mu_{\mathbf{A}_{k}}$ & 2 \\
$\max \mu_{\mathbf{A}_{k}}$ & 82 \\
\hline $\min \sigma_{\mathbf{A}_{k}}^{2}$ & 0.000000 \\
$\max \sigma_{\mathbf{A}_{k}}^{2}$ & 0.333333 \\
\hline $\min \mu_{\mathbf{A}_{k} \text { norm }}^{\text {nax }}$ & 1010.276078 \\
$\max \mu_{\mathbf{A}_{k}^{n o r m}}^{\text {norm }}$ & 2901.018712 \\
\hline $\min \left(\sigma^{2}\right)_{\mathbf{A}_{k} \text { norm }}^{\text {nox }}$ & 0.000002 \\
$\max \left(\sigma^{2}\right)_{\mathbf{A}_{k}}^{\text {nor }}$ & 0.049325 \\
\hline$\mu$ & 42.178899 \\
$\sigma$ & 19.057136 \\
\hline
\end{tabular}

Fig. 12 Statistics computed on the population $\mathcal{P}_{\text {grad }}$ for the utricule and for the saccule. The superindex norm means that the statistics have been computed taking into account the norm of the reference vectors $\mathbf{V}_{\mathbf{A}_{k}}^{d}$ and the vector $\mathbf{A}_{k}$ instead of their direction.

\subsection{Decoding by learning}

We considered a neural network to which are provided $N_{s}$ training examples. Each training examples is a pair $\left(\mathbf{p}^{k}, \mathbf{t}^{k}\right)$, where $\mathbf{p}^{k}$ is a pattern vector of activities and $\mathbf{t}^{k}$ is the corresponding target vector with $k \in\left[1, N_{s}\right]$. Let $n_{p}$ be the number of elements of each pattern vector and $n_{t}$ be the number of elements of each target vector. In our model $n_{p}$ corresponds to the cardinality of the set $\mathcal{P}_{\text {grad }}$, that is $n_{p}=770$ for the utricle and $n_{p}=563$ for the saccule, and $n_{t}$ corresponds to the components of the acceleration vector, that is 2 in a spherical coordinate system. Each element $p_{m}^{k}$ of an input vector corresponds to the response of the neuron $m$ to the corresponding target vector $\mathbf{t}^{k}: p_{m}^{k}=$ $R\left(a_{m}, \mathbf{t}^{k}\right)=R\left(a_{m}, \mathbf{A}_{k}\right)$. Therefore each training example is a pair having the form: $\left(\left[R\left(a_{1}, \mathbf{A}_{k}\right), \ldots, R\left(a_{n_{p}}, \mathbf{A}_{k}\right)\right],\left[\theta_{k}, \phi_{k}\right]\right)$, where $\left(\theta_{k}, \phi_{k}\right)$ are the components of $\mathbf{A}_{k}$. Let $\mathbf{w}_{1}^{n_{p} \times n_{h}}$ be the matrix of synaptic weights between the neurons of the input layer and the neurons of the hidden layer and $\mathbf{w}_{2}^{n_{h} \times n_{t}}$ be the matrix of synaptic weights between the neurons of the hidden layer and the neurons of the output layer.

We stopped the learning algorithm when the average quadratic error between inputs and outputs attained a fixed threshold $\epsilon=10^{-5}$.

Once obtained the matrices of learnt weights $w_{1}$ and $w_{2}$, an estimate $\hat{\mathbf{A}}$ of the stimulus $\mathbf{A}$ can be obtained by using the following formula:

$$
\hat{\mathbf{A}}=\sum_{l=1}^{n_{t}} \Psi\left(\sum_{n=1}^{n_{h}} w_{2}[n][l] \Psi\left(\sum_{m=1}^{n_{p}} w_{1}\left[a_{m}\right][n] R\left(a_{m}, \mathbf{A}\right)\right)\right),
$$

where $\Psi$ denotes the arc-tangent function. We have tested the goodness of the learnt weights over the entire set of accelerations in $\Omega^{\prime}$ and we have evaluated the outcome by calculating the angle between the vectors $\mathbf{A}$ and $\hat{\mathbf{A}}$. We found that the average error for a trial is less than one degree. 
In the region where the slope of the arc-tangent function can be approximated to 1 , we can re-write equation (28) in linear form as:

$$
\hat{\mathbf{A}}=\sum_{l=1}^{n_{t}} \sum_{n=1}^{n_{h}} w_{2}[n][l] \sum_{m=1}^{n_{p}} w_{1}\left[a_{m}\right][n] R\left(a_{m}, \mathbf{A}\right) ;
$$

and we can rewrite this equation as:

$$
\hat{\mathbf{A}}=\sum_{m=1}^{n_{p}} \mathbf{A}_{m} R\left(a_{m}, \mathbf{A}\right),
$$

where $\mathbf{A}_{m}=\sum_{l=1}^{n_{t}} \sum_{n=1}^{n_{h}} w_{2}[n][l] w_{1}\left[a_{m}\right][n]$ represents a new adapted preferred direction associated to the afferent cell $a_{m}$.

4.4 Testing the robustness with respect to neuronal noise

To test the robustness of our model with respect to neuronal noise, at each iteration of the BPNN algorithm, we have sampled the value of the response $\tilde{R}\left(a_{m}, \mathbf{A}_{k}\right)$ of each afferent $a_{m}$ to the acceleration direction $\mathbf{A}_{k}$ from a gaussian noise distribution with standard deviation $\sigma=0.1$ and centered at $\mu=$ $R\left(a_{m}, \mathbf{A}_{k}\right)$ :

$$
\tilde{R}\left(a_{m}, \mathbf{A}_{k}\right)=\frac{1}{\sqrt{2 \pi \sigma^{2}}} \exp \frac{(q-\mu)^{2}}{2 \sigma^{2}},
$$

where $q$ is a random value in the range $[0,1]$. Experimental simulation have proved that the network is able to learn the right weight in presence of neuronal noise so that the error is still of the order of five degrees.

\subsection{Comparison}

In Fig. 13 (right) are shown the functional polarization vectors measured experimentally by Fernandez and Goldberg [16], as well as the distribution of morphological polarization vectors of the hair cells computed by the model of Jaeger et al. [29] for the utricule (up) and for the saccule (down). Before comparing them to the domain of acceleration directions we obtained (see Fig. 13 (left)), we recall that the experimentally recorded afferent vectors include all type of afferents, calyces, dimorphic and boutons, that are distributed along the overall macula. In addition, the simulated density of morphological polarization vectors includes type I and type II hair cells, distributed along the overall macula. On the contrary, the results shown in Fig. 13 (left) have been obtained by modeling only calyces striolar afferents. In principle, our results, which relate directly to afferents, should be compared with the data of Fernandez and Goldberg, more than to the simulations of Jaeger et al., which 
considered single hair cells responses, rather than integrated afferent responses. We found a big similarity between our computational results and the experimental results of Fernandez and Goldberg, and a large overlap with Jaeger et al. modeling results. The dissimilarities can easily be explained. As stated in section 4.2.2, the fact that we have taken into account only the morphological polarization vectors along the striola (see Fig.1) explains why we find functional polarization vectors only on the upper hemisphere for the saccule and only on the left part of both upper and low hemisphere for the utricule. Taking in account the observation of Songer (see [14]), we would have to symmetrize the domain for the saccule with respect to the center of the sphere.

The observed similarity is a non-trivial result, since Fernandez and Goldberg registered afferents from all the maculae surfaces. Thus this confirm our suggestion that striolar and extrastriolar regions cover two similar head acceleration domains, but at different orders of dynamics.

\section{Discussion}

In the present study we obtained an explanation of the three dimensional shape of the striola on the otolith maculae and we predicted the range of linear acceleration directions, including gravity, whose variation is well detected by the striolar system. Our model was based on hypotheses about the function of striolar type I hair cells, about the way afferent cells collect information from them, and about the function of the afferent population. However, this theoretical study and its computational test were based on several simplifications that we discuss in this section, in the light of previous experimental data and computational models.

The main line of thought we followed was that the function of the striolar region is to give maximum possible information about the most dynamic movements of the head, that is rapid, phasic and high frequency information. By essence this information is three dimensional but it has to come from specific morphology and physiology of cells concentrated in a narrow region. Therefore cells from distinct places of the striola have to share their information. The starting hypothesis was that striolar afferent neurons, either dimorphic or calyces, choose their preferred acceleration direction according to the intersection of domains chosen by their calyces. We suggested that one afferent counts for two hair cells in the mean, and detects acceleration by intersection of the preferred planes of the contacted hair cells. This is a doubly non-linear process: type I hair cells must have a non-linear tuning, not cosine but plateau, and afferents must react to as a product, not as a sum or a mean of hair cells. In this model, the effective dimension of the whole striolar system is not one, as would predict a narrow band, but it is two, as if it were a supplementary macula surface. 
UTRICULE
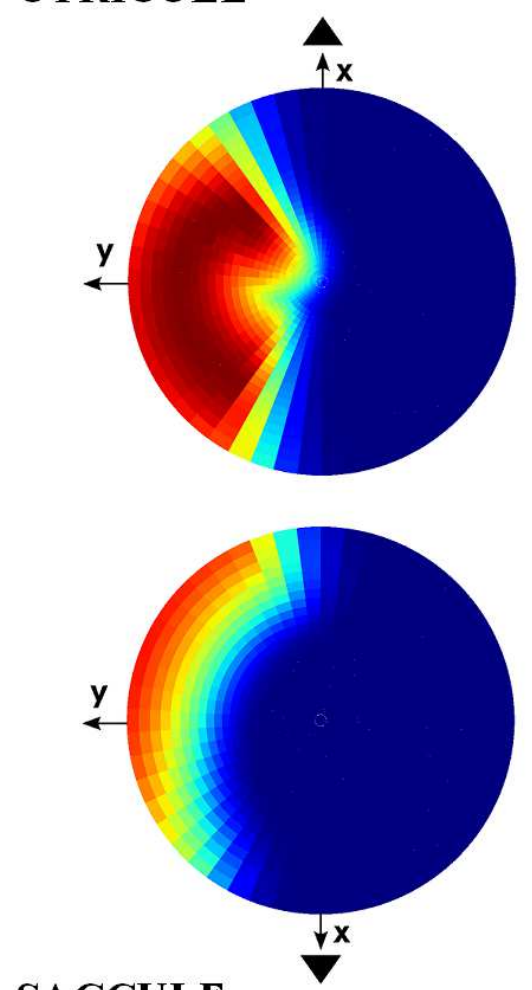

SACCULE
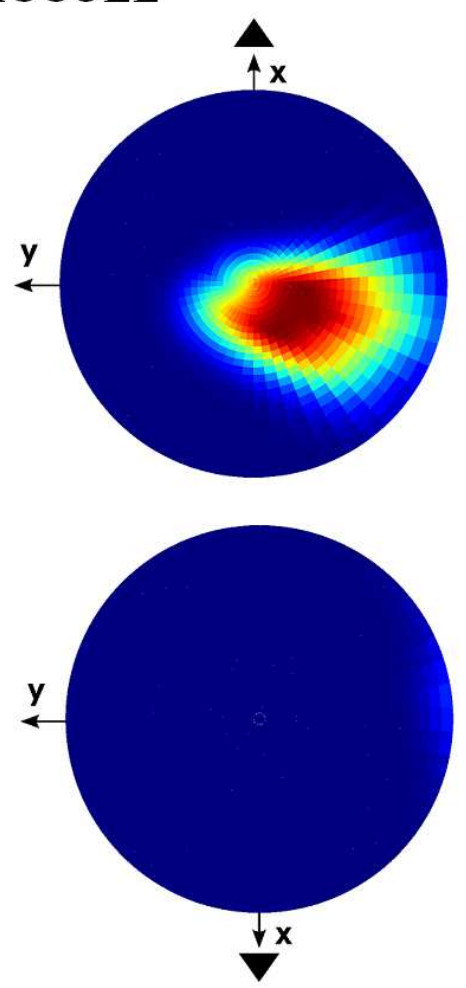
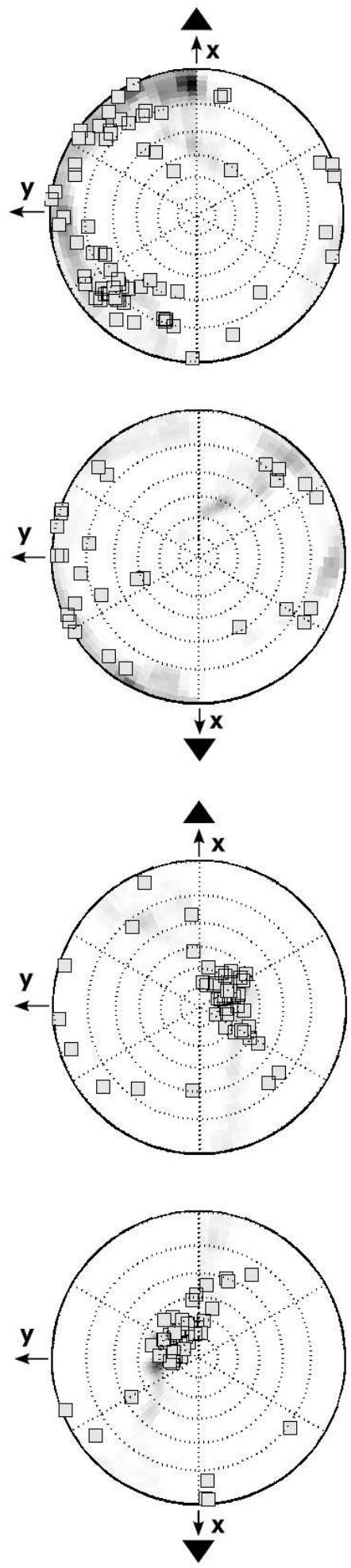

Fig. 13 In this image are shown the stereographic projections of the unit sphere, with the upper hemisphere in the upper row and the lower hemisphere in the lower row. In the left image, for the utricule as well as for the saccule, is shown the maximum value of the response over all selected afferents for each acceleration direction, normalized with respect to the maximum absolute value. In the right image, for the utricule as well as for the saccule, is shown the density of the morphological polarization vectors along the macula modelized by Jaeger et al. [29]. They increase from white over gray to black and were normalized with respect to the largest density found on one of the epithelia. Superimposed on the projections,are also shown polarization vectors found experimentally in single cell recordings from the vestibular nerve of squirrel monkeys by Fernandez and Goldberg [16]. 
Then we can conjecture that sudden acceleration direction changes provoke a shape of activation along the striola with several maximum points (in average two). This is supported by the model of Jaeger and Haslwanter [28]: "... peak responses occur simultaneously on different locations of the striola." We do not exclude the possibility that different processings happen in the saccule and in the utricle (Ross et al. [56], [53]), but we suggest a common geometric principle for acceleration and jerk detection in a large domain.

In addition our model gives three testable predictions:

The first prediction deals with the biophysical properties of the transduction. We predict that the reaction of the type I hair cells in the striolar region is not well fitted by a cosine tuning of the angle with the polarization vector. More precisely, we suggest that a stimulation exerted transversally to the plane of symmetry of the hair bundle (i.e. the plane which is generated by the polarization vector and the normal to the macula), generates a rapidly decreasing reaction, giving zero at an angle less than 90 degrees. On the contrary, a stimulation exerted transversally to the macula generates a cosine decreasing of the reaction of the hair cell.

The second prediction deals with the neural signals in the vestibular nerve. We predict that the afferent neurons on the striolar region react non linearly to the input of the type I hair cells they contact. The exact form of the receptive field would be given by a multiplicative formula of the reactions of the hair cells it connects (as a probability of independent events). This hypothesis could be tested by neurophysiological recordings. For the moment we do not know any direct evidence supporting this prediction.

The third prediction concerns the information flow in the afferent vestibular nerve. We predict that the striolar afferents sense a domain of acceleration as large as the domain sensed by the extrastriolar afferents. However, at least for the utricle, where only one polarization vector occurs along the striola, the receptive field is unilateral, i.e. only medio-lateral excitation occurs for the striolar afferent system. Note that the four striolae together construct a fairly complete mapping of the acceleration domain (see 7). Amazingly, there is a blind angle directed downward, which was also present in the data of Fernandez and Goldberg.

Several existing results help to justify the assumption we made on the hair cells: first, the specificity of striolar bundles compared to extrastriolar bundles was established by Peterson et al. for turtle's utricle, [43], [57], [58], [73]). In particular the type I cells in the striolar region have more numerous and thicker stereocilia, with steeper slope, smaller ratio of kinocilium height over highest stereocilium height (KS), making them adapted to higher frequency tuning. In addition these cells have particularly wide bundles in the direction of the tangent to the striola, i.e. parallel to the PRL. These properties were confirmed in rodents by $\mathrm{Li}$ et al. [35]. According to [38], in mammals, the striolar bundles are wing shaped with more stereocilia along the axis orthogonal to the sensitivity axis, but bundles of peripheral cells are more round and 
compact. Phasic responses are expected for these wider hair bundles, which accords with a broader detection of acceleration directions. Our assumption on the hair cell receptive field are justified by the simulation results of Nam et al. [47]: using parameters of striolar hair cells bundles, in particular transversal wideness, the authors found a plateau around the best response and a nonlinear sudden decreasing of the depolarization of the hair cell for transversal stimulations. Of course, to confirm the stability of our model we have to test a more general non-linearity than $\sin ^{8}(\alpha)$, but the mentioned studies give a direct justification of our assumption. However our precise hypothesis that the striolar type I hair cells detect a plane more than a direction has to be directly verified in situ.

The fact that the receptive fields of hair cells are large in the striola is compatible with the rapid reaction of the striolar system.

The reported afferent cell receptive fields do not have the same kind of nonlinear properties: on the contrary, a non-zero response of afferent neurons is observed for transversal stimulations, 15/100 according to Fernandez and Goldberg [16], [17], [18], and from 4/100 for regular to 8/100 for irregular afferents, in gerbil [13], or pigeon [60]. Rowe and Peterson [58] discussed the origin of this behavior and proposed the hypothesis that the residual response comes from the diversity of the preferences of the contacted hair cells. Note that the majority of afferents have bouton contacts with type II hair cells in addition to their calyces, so that the enlargement of their receptive field could come from these type II cells as well as from type I cells.

The central functional hypothesis of our approach is that multiple calyces allow an afferent neuron to "multiply the reaction of hair cells", as do a coincidence detection in the spatial domain or in the temporal domain. The idea is that along the striola there is more statistical independency among hair cells integration, which accords with the large distances between these cells and the distribution of small otoconia above the striola [72], resulting in a multiplicative joint distributions for the activity of the afferent cells.

Thus we suggest that striolar afferents proceed by elimination, intersecting a set of incomplete sources of information, primarily coming from type I hair cells that are biased to detect planes of directions more than individual directions. We can say that afferent neurons signal a probability of acceleration (or acceleration change) proportional to the product of the individual probabilities of connected hair cells. This could make them bayesian estimators of motion from conditionally independent sources: $P\left(\mathbf{A} \mid H_{1}, H_{2}, \ldots\right)=$ $P\left(H_{1} \mid \mathbf{A}\right) P\left(H_{2} \mid \mathbf{A}\right) \ldots P_{0}(\mathbf{A}) / P(H)$, where the variable $\mathbf{A}$ denotes the acceleration direction, the variable $H=\left(H_{1}, H_{2}, \ldots\right)$ denote the set of hair cells responses, and $P_{0}(\mathbf{A})$ denotes the a priori probability on the acceleration. The denominator $P_{0}(\mathbf{A})$ is necessary in the above Bayes formula; it is the total probability on the stimulus $\mathbf{A}$. The a priori probability could be uniform, but a much more interesting assumption is that the efferent system and the type II hair cells modulate this a priori knowledge. For a recent discussion of the 
application of Bayes inference to neural systems see [71].

The hypothesis that the afferent population detects the variation of acceleration direction as uniformly as possible, corresponds to the maximization of the information on the change of movement direction or gravitation direction.

Known results (see [22] and [14]) support the fact that striolar afferents are efficient for a dynamical detection, transmitting a signal between the jerk and the acceleration of the head. To effectuate such a derivative in time we hypothesized a kernel represented by a Riemann-Liouville integral, i.e. a fractional derivative of a delta function (cf. equation (17) in section 3). Thus we assume that the neurophysiology of afferent cells, probably helped by calyces synapses, is able to reverse the integration into a differentiation. A convincing argument for this derivative function comes from the results of Highstein et al. [26] on the afferent neurons to the cristae of semi-circular canals, where it was established that the gain and the phase both increase with the frequency of the stimulation for frequency after $5-10 \mathrm{~Hz}$, which cannot be due to the biomechanics of the semicircular canals and the cupula [50]. Thus this effect is probably due to the physiology of hair cells and afferent cells.

Ross et al. [55] have investigated the morphological basis for directional sensitivity of vestibular afferents receiving several hair cells. They agreed with Tomko et al. [68] to discard a simple averaging process. More recently Ross et al. [56] have elaborated a three dimansional finite volume model of calyces and ribbon synapses to study the effect of changing geometry of calyces, location and number of synapses, directional input, and activation timing. This computational tool could be used to verify our assertion. More generally it is evident that a more elaborate three dimansional computation is needed to give a better test of our model and its robustness.

In our computational study we have considered type I hair cells perfectly arranged along a curved line, and afferent cells contacting exactly two hair cells. In reality, the striolar region is extended, the polarization vectors of the hair cells have a large variability, and the afferents in their majority contact also type II cells, moreover some afferents enclose only one hair cell, and some others enclose three cells or more. How can we manage this variability and this complexity?

Note that, in our model, each place along the striola curve counts for several type I cells, because it represents as many hair cells the selected afferents contacting this place incapsulate. Thus the striola curve in our model represents a narrow band around the striola on the macula.

Although it would be important to introduce more variability in our model, for example using a random processing in place of a simple geometric model, in the present study we do not add any detail that could by itself have strong correcting effects.

We can expect that the complexity of the biological system preserves the sim- 
ple principles and augments the information flow.

\subsection{Limits of our model}

First, the division of the striolar region on the saccule by the PRL (mentioned in [14]), seems to be contradictory with the assumption of continuity of the polarization. However, in birds the striola possesses this kind of structure on both the utricle and the saccule, but the studies of Dickman et al. [61], [74], show that in this case the afferent neurons contact hair cells on the same side of the PRL, and that the true striola is made of two components.

Second, the majority of striolar afferents contact type II and type I hair cells, but we never have used this overlapping in our model. It could be possible that type II hair cells are responsible of a better precision in sensing the preferred direction of the afferent neurons. Also they can participate in the regulation of $g_{K L}$ conductances which is necessary for the activation of synapses in calyces. Another natural idea for the contribution of type II cells to the information processing along the striola is that that they could be very helpful to take into account the intensity of the sensed acceleration vectors. In fact, in our model we have only considered acceleration directions, in particular it was the only element considered for population selection, but the intensity should be considered in any functional test.

Third, the irregular dynamics of the striolar afferents is an essential property for the vestibular information flow, but we did not model it, as it could be (see [63]). The true selection of a subpopulation for jerk detection should be done by including the dynamics. Thus more computational work has to be done to obtain a complete proof of our model. A complete model should take into account hair cells bundles and polarization variability, the complex geometry of the striolar region, the modeling of calyces terminations, the ion channel kinetics of the hair cells and of their afferents, the modulation by type II cells and the propagation of spike trains along the afferent axons.

\subsection{Phasic and tonic information}

Our model fits well with the hypothesis of the existence of a phasic, irregular, high frequency adapted, striolar sensory subsystem, responsible for linear jerk detection and short latency vestibular information processing.

As otolith end organs and semi-circular canals conjugate their message in the vestibular nuclei, the striolar system information must be combined with a corresponding canal afferent subsystem. Such a subsystem was described in the center of the crista ampullaris, with comparable physiological characteristics although not identical, responsible for detecting rotation acceleration (see $[16],[17][18])$. 
However, the set of afferent dendrites contacting otolith maculae and canals cristae forms a complex parallel processing of presynaptic micro-circuits, with type I terminations regulating type II dendrites, as explained by Ross ([52], [54]). Thus the irregular subsystem must not be confounded with the subsystem of pure calyx afferent neurons. In particular, in mammals it has been shown that type I hair cells are found everywhere on the maculae and on the cristae (see [37]), and that dimorphic connections, mixing calyces and boutons, are largely dominant. In fact, the dynamical properties of phasic-irregular afferent neurons, having a gain which augments with the frequency, depend more on the position of their projections with respect to the macula than on the type of contact (see [19]). This is true for otoliths and for semi-circular canals.

Besides, it was observed that irregular and regular afferents of otoliths and of canals do not generate completely separate flows in the central vestibular organs (see Boyle et al. [3], Peterson [48], Goldberg [21]). Their projections overlap considerably in the vestibular nuclei. They both contribute to the vestibulo-ocular reflex (VOR) and vestibulo-collic reflex (VCR), that stabilize gaze and head respectively, although the irregular input is more involved in the VCR than in the VOR and the reverse is true for the regular input (see [42], [3]). In frogs, phasic and tonic activities in the vestibular nuclei are segregated, but this could be not strictly the case in mammals (nor amniotes) (see $[66],[15]$ ). However, the phasic primary vestibular information of otoliths and canals)can generate short latency responses, participating to synchronization in motor control, posture and autonomic modulation. For instance, the jerk information on translation is implicated in early ocular compensation (compensatory nystagmus), [5], [25], or in subjective vertical assignment [39]. For birds, experiments of Jones et al. [30] on chicken described a net jerk intensity signal, whereas for mammals [32] a fractional order jerk signal (between acceleration and jerk) is reported.

The suggestion we have deduced from our present model of the striola, namely that phasic and tonic vestibular afferents cover the same geometrical fields but at different dynamical and frequency domains, is in favor of overlapped information flows between phasic and tonic pathway, but where different contexts should decide of different ranges of contributions of these pathways for integrated adaptation.

\subsection{Evolution of the striola complex}

Our model can have also an interest from the point of view of evolution. It does not apply to most fishes but it applies to mammals and only partially to birds.

During the evolution of amniotes, the contact with the earth has generated a variety of somato-sensory and proprioceptive information sources to control posture and motion. However, during rapid locomotion the instability of transient contacts makes the somato-sensory information difficult to be 
managed. Consequently it can be expected that vestibular organs of amniotes shifted their sensitivity to more dynamical parameters. This could justify the existence of a subsystem specialized in controlling high order motion likely by sensing acceleration derivatives, thus providing a fundamental capacity for anticipation. (cf. Berthoz et al. [1]). A comparative study between lamprey, rabbit and cat has shown that from the first to the two others there is a shift from vestibular to somatosensory information for postural control (Deliagina et al.[11]). It is possible that an already existent system was adapted for such a new function.

In fact, the presence of the striola has been observed on the otolith maculae of most vertebrate species, with a variety of forms (cf. Lu and Popper [40]), and the heterogeneity of hair cells in vestibular sensors is as old as vertebrates. Even today lampreys have different kind of hair cells on their maculae and cristae. For more recent species under water, the cichlid fishes, Chang et al. [6] and Lanford et al. [31] discovered a distinct type of hair cells along the striola, named type I-like, suggesting that other hair cells on the macula are homologous to type II cells in mammals. This indicates that a functional division on the macula is not an exclusivity of amniotes. However, several facts indicate that the striola in cichlids and mammals has different functions: calyces exist only in amniotes (with rare exceptions on the crista of fishes), efferent neurons attain only type-I like cells in fishes, but in amniotes they attain all types. Moreover type-I like cells of fishes reverse their polarity on the striola curve, that is the PRL coincides with the striola. In mammals, the polarization of the hair cells along the striola varies continuously and this specificity has been important for our model.

Remarkably, in many vertebrates, including amniotes except mammals, there exists an important additional otolith end organ, the lagena, which is also supposed to serve vestibular function. But the arrangement of hair cells polarity along the striola of the lagena is different than the one we have discussed in this manuscript. The polarity is parallel to the curve. There is also a macula neglecta, which, even if small in size, is suspected to provide information about the derivative of angular accelerations [4].

It is amazing to observe that on the saccule of the dipnoi, which is the closest extant fish to tetrapodes, the striola has the standard observed $S$ shape, that has curvature and torsion [49].

In birds, the striola on the utricle, saccule and lagena, appear much more complex than in reptiles and mammals. On the utricle, for instance, the striola has a medial prolongation, where the hair cells are differently organized and oriented, and along the main part of the curve, there is a line of type II hair cells, enclosed by two lines of type I hair cells. Moreover, the otolith afferents seem to detect the jerk on all the macula surface, and it seems that all these afferents are phasic and phase advanced [61]. The increased complexity of the striola structure in birds could reflect a new functionality, such as flying. Thus our model applies only to a part of the striola of birds. We plan to investigate 
the function of the striola in birds in a future study.

\section{Acknowledgements}

This work was supported in part by the European Projects CLONS (FP7ITC-2007.8.0. Project 225929). We are very grateful to Dr. Rudi Jaeger and Professor Emeritus Gerald Jay Goldberg to give us the permission of using their data and reproducing their images. D.B. thanks Ruth-Anne Eatock for very interesting discussions. The authors thank warmly the two anonymous referees which helped them to improve their work and its presentation.

\section{Mathematical appendix}

This appendix provides the mathematical definitions, theorems and proofs, underlying our Striola model. In particular it describes the virtual surface generated by a twisted curve in the Euclidian space and the optimal shapes of curves for a uniform detection of acceleration directions.

The suggested information processing of the striolar region on the otoliths macula lays on the correspondence between the pairs of points of a space curve and the directions orthogonal to the tangents in the points. The shape of the striola is dictated by the range and the regularity of this correspondence, thus we will conduct a study of its properties. In the section 1 we show that the regularity depends on the existence of curvature and torsion. This gives an elementary introduction in the affine framework to the more sophisticated investigations of section 2 and 3. In these following section we compute the optimal form of a striola, if we define optimality by the uniformity of the correspondence, measured by the uniformity of the jacobian determinant of the correspondence. This also corresponds to the uniformity of the representation of variations of acceleration, as considered in the computational model. In section 2 we consider free curves in Euclidian space, in section 3 we consider spherical curves. The reason to restrict to spherical curves is that we represented the macula surface by a spherical lens for optimal detection of the acceleration direction in space by invariant vector fields on the epithelium.

Notations: we consider a smooth curve $S$ in the three dimensional Euclidian space, and we study the correspondence between the pairs of points $\left(P_{1}, P_{2}\right)$ of $S$ and the directions $D$ that are orthogonal to $S$ in $P_{1}$ and $P_{2}$. We are particularly interested in the case where this correspondence defines a mapping $D=F\left(P_{1}, P_{2}\right)$ for pairs of points $P_{1}, P_{2}$ that are sufficiently closed in $S$. We will consider several natural measures of area on the set of pairs of points on $S$ and on the set of directions in the space. The regularity and uniformity of the mapping $F$ is encoded by its jacobian determinant $J$, then we will study 
the link between $J$ and the geometry of $S$. From the Euclidian point of view it is natural to retain on the set of points pairs, the measure associated to the canonical coordinates $\left(\sigma_{1}, \sigma_{2}\right)$, where $\sigma_{1}=s_{1}+s_{2}$ and $\sigma_{2}=s_{1} s_{2}$ are the elementary symmetric functions of the curvilinear abscissas $s_{1}, s_{2}$ of the respective points $P_{1}, P_{2}$ on $S$, and on the set of directions $D$ the measure given by solid angle; this is the framework of section 2 . In section 3 we restrict our attention to the case of a spherical curve $S$, for application to our model of the striola curve. However we begin in section 1 with the Cartesian point of view, where computations are more elementary. In this case a natural parametrization for points on $S$ comes from the orthogonal projection on one axis and natural coordinates for directions in the space come from the intersection with a fixed plane.

\subsection{Cartesian optimal striola}

Let us consider a space curve $S$ described in parametric form by cartesian coordinates

$$
x=f(t), \quad y=g(t), \quad z=h(t) .
$$

If a vector $\vec{A}$ has coordinates $u, v, w$, its scalar product with the tangent $\vec{T}$ of $S$ is given by

$$
\vec{A} \cdot \vec{T}=f^{\prime}(t) u+g^{\prime}(t) v+h^{\prime}(t) w .
$$

The orthogonality equation $\vec{A} \cdot \vec{T}=0$ gives

$$
f^{\prime}(t) u+g^{\prime}(t) v+h^{\prime}(t) w=0 .
$$

We suppose that $\vec{T}$ is nowhere 0 , and that there exist a conical domain of $\vec{A}$ where the equation (34) has two solutions $t_{1}(u, v, w), t_{2}(u, v, w)$ (eventually equals). In this case, for each $\vec{A}$ in the domain, there exist two parameters $t_{1}, t_{2}$ and a non-zero real number $c$ such that

$$
c \vec{A}=\vec{T}\left(t_{1}\right) \times \vec{T}\left(t_{2}\right)
$$

which translates in coordinates as

$$
\begin{aligned}
c u & =g^{\prime}\left(t_{1}\right) h^{\prime}\left(t_{2}\right)-g^{\prime}\left(t_{2}\right) h^{\prime}\left(t_{1}\right), \\
c v & =h^{\prime}\left(t_{1}\right) f^{\prime}\left(t_{2}\right)-h^{\prime}\left(t_{2}\right) f^{\prime}\left(t_{1}\right), \\
c w & =f^{\prime}\left(t_{1}\right) g^{\prime}\left(t_{2}\right)-f^{\prime}\left(t_{2}\right) g^{\prime}\left(t_{1}\right) .
\end{aligned}
$$

A classical theorem asserts that any smooth function $\varphi$ (resp. polynomial function) of two real variables $t_{1}, t_{2}$ (defined in a domain of the plane which is symmetric with respect to the diagonal $\left.t_{1}=t_{2}\right)$ which satisfies $\varphi\left(t_{1}, t_{2}\right)=\varphi\left(t_{2}, t_{1}\right)$ for any pair $\left(t_{1}, t_{2}\right)$, is equal to a smooth (resp. polynomial) function $\Phi\left(\sigma_{1}, \sigma_{2}\right)$ where $\sigma_{1}=t_{1}+t_{2}$ and $\sigma_{2}=t_{1} t_{2}$ are the elementary symmetric functions of $t_{1}$ and $t_{2}$. 
In addition we can suppose that one of the coordinates $u, v, w$ can be arbitrarily chosen, say for instance $w=1$. Then we get a transformation $F$ from a domain of $\left(\sigma_{1}, \sigma_{2}\right)$ to a domain of $(u, v)$. Let us denote $J\left(\sigma_{1}, \sigma_{2}\right)$ the jacobian determinant of the transformation $F$.

Theorem 1: Let us suppose that $J$ is regular and non-vanishing in the neighborhood of a point $(0,0)$ corresponding to a pair of coincident points, then it exists an Euclidian affine change of coordinates $x, y, z$, where we have $f(0)=0$, $f^{\prime}(0) \neq 0, g(0)=g^{\prime}(0)=0$ but $g "(0) \neq 0$, and $h(0)=h^{\prime}(0)=h^{\prime \prime}(0)=0$ but $h^{(3)} \neq 0$.

In other terms, after an Euclidian affine change of frame, the valuations of the Taylor series of $g$ and $h$ are the same as the ones of the twisted cubic.

Proof: We begin by fixing the $x$ axis in such a way that $f(0)=0, f^{\prime}(0) \neq 0$. We change the parametrization $t$ to have $f(t)=a t$ in a neighborhood of $t=0$. It is easy to show this has no effect on the conclusions of the theorem. We suppose $w=1$. In the considered domain of $u, v$ there exist two solutions $t_{1}(u, v), t_{2}(u, v)$ (eventually equals) of the orthogonality equation, that is:

$$
a u+g^{\prime}\left(t_{1}\right) v+h^{\prime}\left(t_{1}\right)=0, \quad a u+g^{\prime}\left(t_{2}\right) v+h^{\prime}\left(t_{2}\right)=0 .
$$

By solving this linear system we obtain $u, v$ from $t_{1}, t_{2}$ :

$$
\begin{aligned}
& u=\frac{h^{\prime}\left(t_{2}\right) g^{\prime}\left(t_{1}\right)-h^{\prime}\left(t_{1}\right) g^{\prime}\left(t_{2}\right)}{a\left(g^{\prime}\left(t_{2}\right)-g^{\prime}\left(t_{1}\right)\right)}, \\
& v=\frac{h^{\prime}\left(t_{1}\right)-h^{\prime}\left(t_{2}\right)}{g^{\prime}\left(t_{2}\right)-g^{\prime}\left(t_{1}\right)} .
\end{aligned}
$$

On another side, by the implicit function theorem we have

$$
\begin{aligned}
& \left(g^{\prime \prime}\left(t_{1}\right) v+h "\left(t_{1}\right)\right) \frac{\partial t_{1}}{\partial u}=-a, \\
& \left(g^{\prime \prime}\left(t_{1}\right) v+h^{\prime \prime}\left(t_{1}\right)\right) \frac{\partial t_{1}}{\partial v}=-g^{\prime}\left(t_{1}\right),
\end{aligned}
$$

and similar equations for $t_{2}$. Thus the jacobian determinant of the transformation from $t_{1}, t_{2}$ to $u, v$ is equal to

$$
J^{\prime}\left(u, v ; t_{1}, t_{2}\right)=a^{-1}\left(g^{\prime}\left(t_{2}\right)-g^{\prime}\left(t_{1}\right)\right)^{-1}\left(g^{\prime \prime}\left(t_{1}\right) v+h "\left(t_{1}\right)\right)\left(g^{\prime \prime}\left(t_{2}\right) v+h "\left(t_{2}\right)\right)
$$

We remark that where $t_{1}=t_{2}$, the function $J^{\prime}$ has a priori a simple zero $\left(t_{1}-t_{2}\right)$ along the diagonal subset. However the jacobian of the transformation which maps $\left(t_{1}, t_{2}\right)$ to $\left(\sigma_{1}, \sigma_{2}\right)$ is equal to $t_{1}-t_{2}$, so the jacobian of the mapping $F$ in the coordinates $u, v$ and $\sigma_{1}, \sigma_{2}$ is given by the regular symmetric function

$$
J\left(\sigma_{1}, \sigma_{2}\right)=\left[a\left(t_{1}-t_{2}\right)\left(g^{\prime}\left(t_{2}\right)-g^{\prime}\left(t_{1}\right)\right)\right]^{-1}\left(g^{\prime \prime}\left(t_{1}\right) v+h^{\prime \prime}\left(t_{1}\right)\right)\left(g^{\prime \prime}\left(t_{2}\right) v+h "\left(t_{2}\right)\right)
$$

that is in general finite and non-zero along the diagonal.

Let us suppose that $J$ is a smooth function without zero in an open set of 

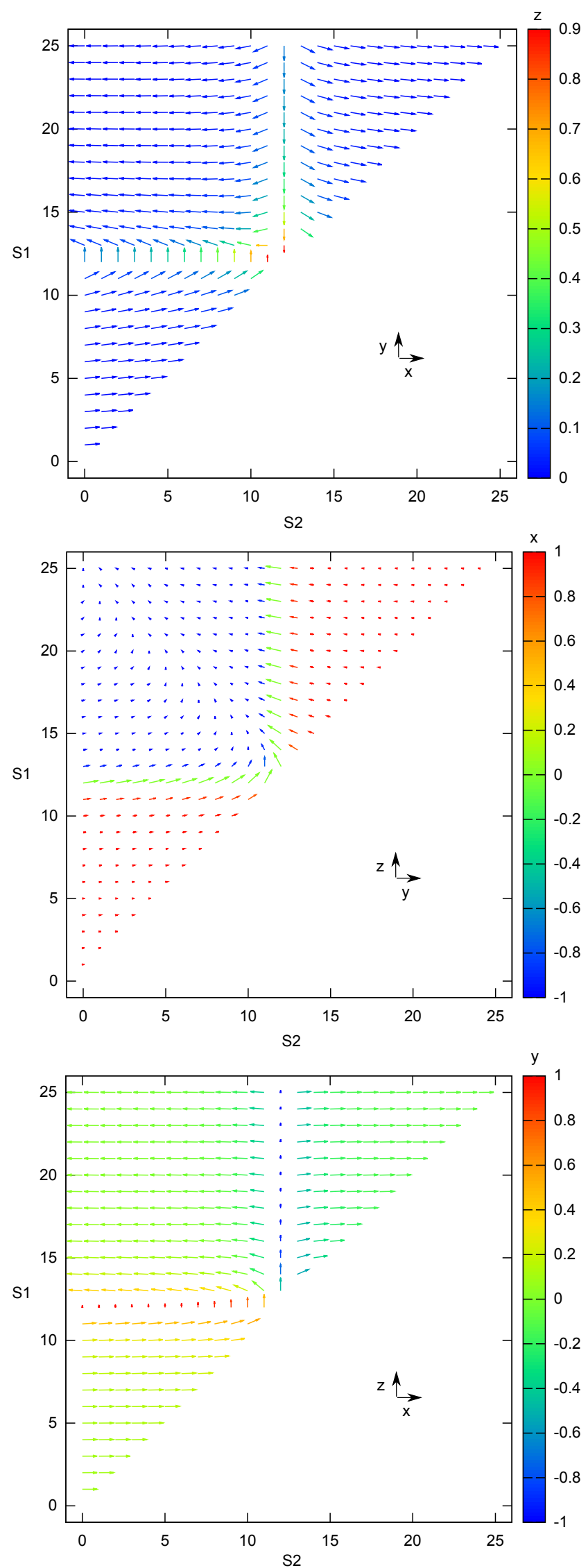

Fig. 14 Representation of the preferred direction encoded by each couples of cells. It is here computed for the curve $x(t)=t / \sqrt{(2)}, y=t^{2}, z=\sqrt{(2) / 3 \times t^{3}}$, for $t \in[-10 ; 10]$, with 25 cells distributed equally along t. Panels show the projection of this surface in the $(x, y)$, $(y, z)$ and $(x, z)$ planes, the color palette represents the value of missing component $(\mathrm{z}, \mathrm{x}$ and y respectively). 
$\left(t_{1}, t_{2}\right)$ containing $(0,0)$. By substitution of the values of $u$ and $v$ we obtain the following functional equation for $g$ and $h$ :

$$
\begin{aligned}
& {\left[g^{\prime \prime}\left(t_{1}\right)\left(h^{\prime}\left(t_{1}\right)-h^{\prime}\left(t_{2}\right)\right)+h "\left(t_{1}\right)\left(g^{\prime}\left(t_{2}\right)-g^{\prime}\left(t_{1}\right)\right)\right]} \\
& {\left[g^{\prime \prime}\left(t_{2}\right)\left(h^{\prime}\left(t_{1}\right)-h^{\prime}\left(t_{2}\right)\right)+h "\left(t_{2}\right)\left(g^{\prime}\left(t_{2}\right)-g^{\prime}\left(t_{1}\right)\right)\right]} \\
& =J\left(t_{1}, t_{2}\right) a\left(t_{1}-t_{2}\right)\left(g^{\prime}\left(t_{2}\right)-g^{\prime}\left(t_{1}\right)\right)^{3},
\end{aligned}
$$

Let us suppose that $t_{1}$ and $t_{2}$ both approach $t$, in such a manner that $t=$ $\alpha t_{1}+\beta t_{2}$ with $\alpha, \beta$ bounded and $\alpha+\beta=1$. Up to the order 4 in $t_{2}-t_{1}$ (or $t_{1}-t$ or $t_{2}-t$ because it is the same order) we have

$$
\begin{gathered}
{\left[\left(\left(t_{1}-t\right)\left(t_{1}-t_{2}\right)+\frac{1}{2}\left(t_{2}-t_{1}\right)\left(t_{1}+t_{2}-2 t\right)\right) h^{\prime \prime}(t) g^{(3)}(t)\right)} \\
\left.\left.+\left(\left(t_{1}-t\right)\left(t_{2}-t_{1}\right)+\frac{1}{2}\left(t_{1}-t_{2}\right)\left(t_{1}+t_{2}-2 t\right)\right) h^{(3)}(t) g^{\prime \prime}(t)\right)\right] \\
{\left[\left(\left(t_{2}-t\right)\left(t_{1}-t_{2}\right)+\frac{1}{2}\left(t_{2}-t_{1}\right)\left(t_{1}+t_{2}-2 t\right)\right) h^{\prime \prime}(t) g^{(3)}(t)\right)} \\
\left.\left.+\left(\left(t_{2}-t\right)\left(t_{2}-t_{1}\right)+\frac{1}{2}\left(t_{1}-t_{2}\right)\left(t_{1}+t_{2}-2 t\right)\right) h^{(3)}(t) g^{\prime \prime}(t)\right)\right] \\
=-a J(t, t)\left(t_{1}-t_{2}\right)^{4} g^{\prime \prime}(t)^{3},
\end{gathered}
$$

We also have $t_{1}-t=\beta\left(t_{1}-t_{2}\right), \quad t_{2}-t=\alpha\left(t_{2}-t_{1}\right), \quad t_{1}+t_{2}-2 t=$ $(2 \beta-1)\left(t_{1}-t_{2}\right)$, then, at the limit, we get

$$
\begin{array}{r}
\left.\left.\left[\frac{1}{2} h^{\prime \prime}(t) g^{(3)}(t)\right)-\frac{1}{2} h^{(3)}(t) g^{\prime \prime}(t)\right)\right] \\
\left.\left.\left[-\frac{1}{2} h^{\prime \prime}(t) g^{(3)}(t)\right)+\frac{1}{2} h^{(3)}(t) g^{\prime \prime}(t)\right)\right] \\
=-a J(t, t) g^{\prime \prime}(t)^{3} .
\end{array}
$$

We would have found the same formula by fixing $t_{1}=t$ and looking at $t_{2}$ tending to $t$.

Let us write $G=g$ " and $H=h "$, we obtain

$$
\left(G^{\prime} H-G H^{\prime}\right)^{2}=4 a J G^{3},
$$

From this we see that $G$ cannot vanish, meaning that $g$ is necessarily convex or concave. By changing the sign of the coordinate $y$ in the ambient space, we can suppose that $g$ is convex, then $G$ is positive and $J$ positive.

By using a translation and a rotation in the plane $x, y$ we can suppose that $g(0)=g^{\prime}(0)=0$, but $g^{\prime \prime}(0)=G(0)>0$, which gives a development

$$
g(t)=\frac{1}{2} b t^{2}+\frac{1}{3} b_{1} t^{3}+\ldots
$$

When $g$ is known as well as the initial condition $H(0)=h "(0)$, the linear differential equation

$$
H^{\prime}=\left(G^{\prime} / G\right) H+2(a G J)^{1 / 2},
$$


determines $H$ as

$$
H(t)=G(t) \frac{H(0)}{G(0)}+2 G(t) \int_{0}^{t} \sqrt{\frac{a J(s)}{G(s)}} d s .
$$

Then, knowing the values of $h(0), h^{\prime}(0)$ in addition to $h "(0)$, we obtain $h$ by two other quadratures.

With a vertical translation we can assume $h(0)=0$, and with a rotation in the plane $x, z$ we can assume $h^{\prime}(0)=0$.

By rotating the plane $y, z$ we can then make $h "(0)=0$.

Now we can apply the formula (52) and the theorem 1 follows.

When $f, g, h$ satisfy the conclusions of the theorem 1 , the axis $x, y, z$ are respectively aligned with the tangent, the normal and the binormal axis of the curve $S$ at the origin point 0 . The curvature $\kappa(0)$ and the torsion $\tau(0)$ of $S$ in 0 are given by

$$
\kappa(0)=\frac{g^{\prime \prime}(0)}{f^{\prime}(0)^{2}}, \quad \tau(0)=-\frac{h^{(3)}(0)}{f^{\prime}(0) g^{\prime \prime}(0)} .
$$

Let us verify by direct computation that every polynomials $g, h$ of respective degree 2 and 3 satisfy the formula (46) for all points $\left(t_{1}, t_{2}\right)$ with $J$ a non-zero constant:

We can write

$$
f(t)=a t, \quad g(t)=\frac{b}{2} t^{2}+b_{1} t+b_{0}, \quad h(t)=\frac{c}{3} t^{3}+c_{2} t^{2}+c_{1} t+c_{0},
$$

then we have

$$
\begin{aligned}
g^{\prime}\left(t_{2}\right)-g^{\prime}\left(t_{1}\right) & =b\left(t_{2}-t_{1}\right), \\
h^{\prime}\left(t_{1}\right)-h^{\prime}\left(t_{2}\right) & =c\left(t_{1}-t_{2}\right)\left(t_{2}+t_{1}\right)+2 c_{2}\left(t_{1}-t_{2}\right), \\
g^{\prime \prime}(t) & =b, \\
h^{\prime \prime}(t) & =2\left(c t+c_{2}\right),
\end{aligned}
$$

then

$$
\begin{aligned}
g "\left(t_{1}\right)\left(h^{\prime}\left(t_{1}\right)-h^{\prime}\left(t_{2}\right)\right)+h "\left(t_{1}\right)\left(g^{\prime}\left(t_{2}\right)-g^{\prime}\left(t_{1}\right)\right) & =-b c\left(t_{2}-t_{1}\right)^{2}, \\
g "\left(t_{2}\right)\left(h^{\prime}\left(t_{1}\right)-h^{\prime}\left(t_{2}\right)\right)+h "\left(t_{2}\right)\left(g^{\prime}\left(t_{2}\right)-g^{\prime}\left(t_{1}\right)\right) & =b c\left(t_{2}-t_{1}\right)^{2}, \\
\left(t_{2}-t_{1}\right)\left(g^{\prime}\left(t_{2}\right)-g^{\prime}\left(t_{1}\right)\right)^{3} & =b^{3}\left(t_{2}-t_{1}\right)^{4},
\end{aligned}
$$

and (46) is satisfied with

$$
\frac{b^{2} c^{2}}{b^{3}}=a J
$$

that is

$$
J=\frac{c^{2}}{a b} .
$$


Theorem 2: Suppose that $f, g, h$ satisfy the conclusions of the theorem 1 , and that $f$ is a linear function of $t$; then $J$ is constant in the neighborhood of the diagonal if and only if $g$ is a polynomial of degree 2 and $h$ is a polynomial of degree 3 .

Proof: Let us fix the constant $J$ and the parameter $t_{2}$; the two equations (46), (49) constitute a system of two non autonomous ordinary differential equations in two unknown functions $g^{\prime}, h^{\prime}$ of order one in $g^{\prime}$ and order two in $h^{\prime}$, so by the Cauchy-Lipschitz theorem, the general solution depends on three independent real parameters when the initial time is chosen, thus the general solution of the system when initial time is let free depends on four real constants. But we already know solutions of the system depending on four real parameters, they are polynomials in $t$ of degree 1 for $g^{\prime}$ and of degree 2 for $h^{\prime}$. CQFD.

Thus, in the case of theorem 1, we obtain a semi-cubic parabola

$$
x=a t, \quad y=\frac{1}{2} a^{2} \kappa(0) t^{2}, \quad z=-\frac{1}{6} a^{3} \kappa(0) \tau(0) t^{3} .
$$

\subsection{Euclidian optimal striola}

The natural measure on directions in a 3D Euclidian space is the solid angle $d \sigma$, and the natural parametrization of a curve is by its arc-length $s$. Therefore it is natural to look at the correspondence between pairs of points and directions in these coordinates. We will first establish a formula for the Jacobian determinant in these coordinates. As before we will constat it has a simple zero. As in the Cartesian study, the symmetric coordinates operate a regularization. But the new ingredient here is the surface obtained by translating the curve by itself, that was considered by Sophus Lie, for application to minimal surfaces. The points on this auxiliary surface form another symmetric parametrization of pairs of points, giving another natural regularization.

Let us introduce a surface $\Sigma$ associated to the curve $S$, it is formed by adding the pair of points of the curve:

$$
\begin{aligned}
& x\left(s_{1}, s_{2}\right)=f\left(s_{1}\right)+f\left(s_{2}\right), \\
& y\left(s_{1}, s_{2}\right)=g\left(s_{1}\right)+g\left(s_{2}\right), \\
& z\left(s_{1}, s_{2}\right)=h\left(s_{1}\right)+h\left(s_{2}\right) .
\end{aligned}
$$

Let us denote by $P_{1}, P_{2}$ the points of abscissas $s_{1}, s_{2}$ respectively on the curve $S$. By fixing $s_{2}$ (resp. $s_{1}$ ) we see that the tangent vector $\vec{T}_{1}\left(\right.$ resp. $\overrightarrow{T_{2}}$ ) to $S$ in $P_{1}$ (resp. $P_{2}$ ) is tangent to the surface $\Sigma$. Then the cross product $\overrightarrow{T_{1}} \times \overrightarrow{T_{2}}$ is normal to $\Sigma$. Thus for generic points, when $\vec{T}_{1}$ and $\overrightarrow{T_{2}}$ are not collinear, the unit normal vector $\vec{n}$ to $\Sigma$ at the point corresponding to $\left(s_{1}, s_{2}\right)$ is parallel to the intersection of the normal planes at $S$ in $P_{1}$ and $P_{2}$. This is the way $\Sigma$ represents the pair of points $\left(P_{1}, P_{2}\right)$ on $S$, by developing their associated 
direction $D$ in the space.

This surface $\Sigma$ is a particular case or a "Translation Surface", in the sense of S. Lie, H. Poincaré, G. Darboux (Leçons sur la Théorie gé né rale des Surfaces et les applications gé omé triques du Calcul Infinité simal; Part I, L.III, ch. VI, VII, VIII, IX; [10]). The general translation surfaces are the surfaces described by the sum of points of two given space curves. In our case, the curve is translated by itself.

Note that the surface which is defined as the locus of middle points of the segments that join the pairs of points of the curve $S$, is the surface homothetic to $\Sigma$ in the ratio $1 / 2$, then it has the same normals and could be used in the place of $\Sigma$ in what follows.

According to Lie, by using imaginary parameters, any minimal surface is described as a translation surface starting from a complex curve with tangent vectors of zero lengths.

Caution: the surface $\Sigma$ does not represent the macula, it represents the directions of acceleration detected by the striola by its normal vectors. It is a kind of virtual macula associated to the striola. The parametrization by $s_{1}, s_{2}$ is singular, because two pairs $\left(s_{1}, s_{2}\right)$ and $\left(s_{2}, s_{1}\right)$ represent the same point, but the surface $\Sigma$ itself is in general regular. Let us see what happens in the example of the surface generated by the twisted cubic; in this case we have:

$$
\begin{aligned}
& x\left(t_{1}, t_{2}\right)=t_{1}+t_{2}, \\
& y\left(t_{1}, t_{2}\right)=t_{1}^{2}+t_{2}^{2}, \\
& z\left(t_{1}, t_{2}\right)=t_{1}^{3}+t_{2}^{3} .
\end{aligned}
$$

We then have

$$
z-x y=t_{1} t_{2}^{2}+t_{2} t_{1}^{2}=x\left(t_{1} t_{2}\right)=\frac{1}{2} x\left(x^{2}-y\right),
$$

so $\Sigma$ is included in the smooth graph of a polynomial function:

$$
z=\frac{1}{2} x\left(y+x^{2}\right)
$$

And it is easy to show that every point $(x, y, z)$ in this graph such that $y \geq x^{2} / 2$ belongs to $\Sigma$. Again the parametrization problem comes from the symmetry between $t_{1}$ and $t_{2}$, but the regular prolongation comes from the fact that every symmetric polynomial of $t_{1}, t_{2}$, like $t_{1}^{3}+t_{2}^{3}$, is a function of $x=t_{1}+t_{2}$ and $y=t_{1}^{2}+t_{2}^{2}$. (This theorem needs rational coefficients, integers are not sufficient are they were for $t_{1}+t_{2}$ and $t_{1} t_{2}$, but this has no consequence in the present context.) As the example of twisted cubic shows, the set of directions that are detected by real parameters $t_{1}, t_{2}$ is the set of normals of a regular surface 
with boundary.

For the general twisted cubic

$$
x=a t, \quad y=\frac{b}{2} t^{2}, \quad z=\frac{c}{3} t^{3}
$$

we find the region $y \geq b x^{2} / 4 a^{2}$ on the gather surface

$$
z=\frac{c}{6 a^{3} b}\left(b x^{3}+2 a^{2} x y\right) .
$$

In what follows, the numbers $s_{1}, s_{2}$ will design arc-lengths on the curve $S$. We denote by $\vec{T}_{1}$ resp. $\overrightarrow{T_{2}}$ the unit tangent to the curve $S$ at $s_{1}$ resp. $s_{2}$. Let us suppose they are linearly independent and denote by $\vec{n}$ the unit normal to the plane they generate, such that $\left(\vec{T}_{1}, \overrightarrow{T_{2}}, \vec{n}\right)$ constitute a right handed oriented reference frame, which corresponds to the vector product formula: $\left|\sin \left(\operatorname{arcos}\left(\overrightarrow{T_{1}} \cdot \overrightarrow{T_{2}}\right)\right)\right| \vec{n}=\overrightarrow{T_{1}} \times \overrightarrow{T_{2}}$.

For any surface in $\mathbb{R}^{3}$ the Gauss mapping is the map to the unit sphere, which sends a point to the unit normal of the surface in this point, thus in our case the Gauss mapping $\gamma$ sends the point of $\Sigma$ corresponding to $s_{1}, s_{2}$ onto the vector $\vec{n}$. This map is specially interesting for us because $\vec{n}$ represents the preferred direction of acceleration of an afferent cell associated to the parameters $\left(s_{1}, s_{2}\right)$.

By a theorem of Gauss, the determinant of the jacobian of $\gamma$ from orthonormal coordinates on $\Sigma$ to solid angle is the Gauss curvature function $K$ of the surface $\Sigma$.

The Riemannian metric on $\Sigma$ has coefficients $g_{i j}$ defined by the scalar products $\vec{T}_{i} \cdot \vec{T}_{j}$, cf. Coxeter, Introduction to Geometry (Wiley, 1969).

In our case $g_{11}=g_{22}=1$. The superficial area measure is given in these coordinates by the formula

$$
d A=\sqrt{g} d s_{1} d s_{2}
$$

where we noted

$$
g=g_{11} g_{22}-g_{12}^{2}=1-\left(\overrightarrow{T_{1}} \cdot \overrightarrow{T_{2}}\right)^{2}
$$

Observe that the two families of curves respectively defined by fixing $s_{1}+s_{2}$ or $s_{1}-s_{2}$ equal to constants, are orthogonal one to each other.

We denote by $g^{i j}$ the coefficients of the inverse matrix of $\left(g_{i j}\right)$; they are

$$
g^{11}=g^{22}=g^{-1}, \quad g^{12}=g^{21}=-g^{-1} g_{12} .
$$

The first Christoffel symbols (describing the intrinsic parallel transport on the surface) are defined as

$$
\Gamma_{i j ; k}=\frac{1}{2}\left(\partial_{i} g_{j k}+\partial_{j} g_{i k}-\partial_{k} g_{i j}\right) .
$$

In our case the only non-zero symbols are

$$
\Gamma_{11 ; 2}=\partial_{1}\left(g_{12}\right), \quad \Gamma_{22 ; 1}=\partial_{2}\left(g_{12}\right) .
$$


The second (and more usual) Christoffel symbols are defined as

$$
\Gamma_{i j}^{k}=\sum g^{k l} \Gamma_{i j ; k}
$$

Thus in our case they are non-zero only if $i=j$. All the non-zero symbols are given by:

$$
\begin{gathered}
\Gamma_{11}^{1}=\frac{-g_{12} \partial_{1}\left(g_{12}\right)}{g}, \quad \Gamma_{11}^{2}=\frac{\partial_{1}\left(g_{12}\right)}{g}, \\
\Gamma_{22}^{1}=\frac{\partial_{2}\left(g_{12}\right)}{g}, \quad \Gamma_{22}^{2}=\frac{-g_{12} \partial_{2}\left(g_{12}\right)}{g},
\end{gathered}
$$

It is useful, in our case, to relate these intrinsic quantities with the parametric representation; for that we introduce the second derivatives vectors

$$
\overrightarrow{T_{i j}}=\frac{\partial}{\partial s_{j}} \overrightarrow{T_{i}}
$$

Then the Christoffel symbols are given by scalar products as

$$
\Gamma_{i j ; k}=\overrightarrow{T_{k}} \cdot \overrightarrow{T_{i j}}
$$

We can also define

$$
b_{i j}=\vec{n} \cdot \overrightarrow{T_{i j}}
$$

They are the coefficients of the second fundamental form of $\Sigma$. In our present case only $b_{11}$ and $b_{22}$ could be non-zero. The corresponding Weingarten coefficients are

$$
b_{j}^{i}=\sum g^{i k} b_{k j}
$$

thus, in our case

$$
b_{1}^{1}=g^{-1} b_{11}, \quad b_{2}^{2}=g^{-1} b_{22}, \quad b_{2}^{1}=b_{1}^{2}=-g^{-1} g_{12} b_{12} .
$$

Then we obtain the "equations of Gauss":

$$
\overrightarrow{T_{i j}}=\sum \Gamma_{i j}^{k} \overrightarrow{T_{k}}+b_{i j} \vec{n}
$$

If we denote by $b$ the discriminant of the second fundamental form

$$
b=b_{11} b_{22}-b_{12}^{2},
$$

the Gauss curvature is defined by

$$
K=\frac{b}{g} .
$$

The Theorema egregium of Gauss says that $K$ can be expressed by the derivatives of the second symbols. A nice formula was found by Liouville (cf. Coxeter p.367):

$$
\sqrt{g} K=\frac{\partial}{\partial s_{2}}\left(\frac{\sqrt{g}}{g_{11}} \Gamma_{11}^{2}\right)-\frac{\partial}{\partial s_{1}}\left(\frac{\sqrt{g}}{g_{11}} \Gamma_{12}^{2}\right)
$$


Note, in our case we have $\Gamma_{12}^{2}=0$, and

$$
\Gamma_{11}^{2}=\frac{\overrightarrow{T_{11}} \cdot \overrightarrow{T_{2}}}{g} .
$$

(This is because $\overrightarrow{T_{11}}$ is orthogonal to $\overrightarrow{T_{1}}$.)

The Gauss map has the curvature as its jacobian determinant:

$$
\frac{d \sigma}{\sqrt{g} d s_{1} d s_{2}}=K
$$

Thus the jacobian determinant from $d s_{1} d s_{2}$ to $d \sigma$ is given by

$$
J^{\prime}=\sqrt{g} K=\frac{\partial}{\partial s_{2}} \frac{\overrightarrow{T_{11}} \cdot \overrightarrow{T_{2}}}{\sqrt{g}}
$$

Which can be rewritten as

$$
J^{\prime}=\frac{\left(\overrightarrow{T_{1}} \cdot \overrightarrow{T_{2}}\right)\left(\overrightarrow{T_{11}} \cdot \overrightarrow{T_{2}}\right)\left(\overrightarrow{T_{22}} \cdot \overrightarrow{T_{1}}\right)+g\left(\overrightarrow{T_{11}} \cdot \overrightarrow{T_{22}}\right)}{g^{3 / 2}}
$$

From the first section we know that $J^{\prime}$ has a simple zero along the diagonal (of the first order like $\left(s_{1}-s_{2}\right)$ multiplied by a constant). This invitees to make a re-normalization of $J^{\prime}$. A natural choice is to divide by $s_{1}-s_{2}$ because this is the jacobian of the transformation from the coordinates $\left(s_{1}, s_{2}\right)$ to the elementary symmetric function $\sigma_{1}=s_{1}+s_{2}, \sigma_{2}=s_{1} s_{2}$. But also $\sqrt{g}$ has a first order zero on the diagonal $s_{1}=s_{2}$, then an alternative natural choice to re-normalize $J^{\prime}$ is to take the curvature $K$ itself. This corresponds to the representation of the set of pairs of points on $S$ by the surface $\Sigma$ itself. With this second choice, the optimality problem becomes: to find the curves $S$ such that the associated double surface of translation $\Sigma$ has the less possible changing Gaussian curvature. We will compute now this curvature $K$, and the density $\sqrt{g}$.

The jacobian of the mapping from affine coordinates $(u, v, 1)$ on directions to the spherical angle measure is given by

$$
J(u, v ; \sigma)=\frac{1}{\sqrt{1+u^{2}+v^{2}}},
$$

where $u, v$ are given in function of $s_{1}, s_{2}$ by the formulas

$$
\begin{aligned}
& u=\frac{g_{1} h_{2}-h_{1} g_{2}}{f_{1} g_{2}-g_{1} f_{2}} \\
& v=\frac{h_{1} f_{2}-f_{1} h_{2}}{f_{1} g_{2}-g_{1} f_{2}}
\end{aligned}
$$

For computation another formula is helpful, coming from Weingarten (see Coxeter [7] p.354):

$$
\sqrt{g} K=\left[\vec{n} ; \partial_{1} \vec{n} ; \partial_{2} \vec{n}\right],
$$


where the bracket denotes the determinant of vectors, or triple product. In fact this Weingarten formula is the origin of the recalled Liouville formula. In our present situation the formula can be rewritten by using vector products as follows:

$$
g^{2} K=\left[\overrightarrow{T_{1}} \times \overrightarrow{T_{2}} ; \overrightarrow{T_{11}} \times \overrightarrow{T_{2}} ; \overrightarrow{T_{1}} \times \overrightarrow{T_{22}}\right]
$$

Theorem 3: let us denote by $\kappa, \tau$ the curvature and torsion of $S$, and $\dot{\kappa}, \dot{\tau}$ their derivatives with respect to the arc-length $s$ on $S$; then along the double curve $S$ on $\Sigma$ we have the limit formula

$$
K=-\frac{\tau^{2}}{4} .
$$

At the first order in $s_{1}-s, s_{2}-s$, we have

$$
K\left(s_{1}, s_{2}\right)=-\frac{\tau^{2}}{4}\left[1+\left(s_{1}+s_{2}-2 s\right)(\dot{\tau} / \tau)\right],
$$

and

$$
g\left(s_{1}, s_{2}\right)=\kappa^{2}\left(s_{1}-s_{2}\right)^{2}\left[1+\left(s_{1}+s_{2}-2 s\right)(\dot{\kappa} / \kappa)\right] .
$$

Proof: we use Taylor formula for evaluating $\overrightarrow{t_{1}}, \overrightarrow{t_{2}}$, and recurrently all terms in the equation (100). (Note that we verified this result by using equation (90).) The proof is a straightforward but tedious computation.

As a corollary we obtain the amazing result that for twisted curves, when $\tau$ has no zero, the Gaussian curvature of the double surface is everywhere finite and strictly negative.

Theorem 3 is a precision of the theorem 1, because it shows directly that a curve giving good vector detection in space must be twisted, but it uses the fact that curvature and torsion are well defined, which is one of the results of theorem 1.

If we choose the surface $\Sigma$ as the natural parametrization of pairs of points $\left(P_{1}, P_{2}\right)$ on $S$, the absolute value of the Jacobian determinant from pairs of points to directions in space is approximatively given by

$$
|K| \approx \frac{1}{4} \tau^{2}\left(1+\frac{d(\ln (\tau))}{d s}\left(\sigma_{1}-2 s\right)\right)
$$

for near points, thus, at the first order of approximation, the optimal curves for uniform detection of directions must have a constant torsion $\tau$. But, if we choose the elementary symmetric functions $\left(\sigma_{1}, \sigma_{2}\right)$ as the natural parametrization of pairs of points $\left(P_{1}, P_{2}\right)$ on $S$, the absolute value of the Jacobian determinant for near points is given by

$$
|J|=\sqrt{g}|K| /\left|s_{1}-s_{2}\right| \approx \frac{1}{4} \kappa \tau^{2}\left(1+\frac{1}{2} \frac{d\left(\ln \left(\kappa \tau^{2}\right)\right)}{d s}\left(\sigma_{1}-2 s\right)\right) .
$$


Thus the optimal curves correspond to constant value of the quantity $\tau \sqrt{\kappa}$.

At higher orders in the formula of $K$ it appears polynomials in the two variables $s_{1}-s_{2}, s_{1}+s_{2}-2 s$ whose coefficients are polynomials in the higher derivatives of $\kappa, \tau$ with respect to $s$. At the order two for instance we obtain two coefficients of the following form

$$
\begin{aligned}
& P(\kappa, \tau, \dot{\kappa}, \dot{\tau}, \ddot{\kappa}, \ddot{\tau})= \\
& \kappa^{2} \tau^{2}\left[A \ddot{\kappa} / \kappa+B \ddot{\tau} / \tau+C(\dot{\kappa} / \kappa)^{2}+D(\dot{\tau} / \tau)^{2}+E(\dot{\kappa} \dot{\tau} / \kappa \tau)+F\left(\kappa^{2}+\tau^{2}\right)\right]
\end{aligned}
$$

In particular, the only possibility that the order two computation of $K$ gives a development $-\frac{\tau^{2}}{4}\left[1+\alpha\left(s_{1}-s_{2}\right)^{2}\right]$ with a constant $\alpha$, is that $\kappa$ and $\tau$ are constants. That is $S$ must be a circular helix.

The standard circular helix in the vertical cylinder of radius $a$ is defined as

$$
x=a \cos t, \quad y=a \sin t, \quad z=c t .
$$

One has

$$
\begin{gathered}
\kappa=\frac{a}{a^{2}+c^{2}}, \quad \tau=\frac{c}{a^{2}+c^{2}}, \\
a=\frac{\kappa}{\kappa^{2}+\tau^{2}} .
\end{gathered}
$$

(cf. Coxeter pp.323-325). The principal normal $\vec{p}=\dot{\vec{T}} / \kappa$ is always horizontal and its binormal $\mathbf{b}$, as its tangent $\vec{T}$, makes a constant angle with the vertical. This binormal vector $\vec{b}$ is important for us because it is the limit for coincident points $P_{1}, P_{2}$ of the normal vector $\vec{n}$ to the surface $\Sigma$.

In the case of helix we have the following formula for the curvature of $\Sigma$ :

$$
K=-\frac{\tau^{2}}{4}\left[1+a \frac{\kappa}{2}\left(\cos \left(u_{1}-u_{2}\right)-1\right)\right]^{-2} .
$$

In perception, some acceleration directions $\vec{A}$ could be more natural than others. But, on directions in space, the 2D spherical Von-Mises distributions have maximum entropy among the laws when mean and variances are given; then it would be interesting to look at curves giving approximatively such laws for the preferred directions.

There is also a notion of generalized helix (cf. Coxeter p.325). They are the curves where the ration $\tau / \kappa$ has a constant value. They are characterized by the fact that their tangent vectors make a constant angle with a fixed vector. The standard twisted cubic is not a circular helix, but a linear transform of it is a generalized helix, that is $\tau / \kappa$ is a constant, and it has near its vertex a torsion and a curvature which are stationary. In fact let us consider the following special twisted cubic:

$$
x=\frac{t}{\sqrt{2}}, \quad y=t^{2}, \quad z=\frac{\sqrt{2}}{3} t^{3} ;
$$


then its curvature and torsion are given by

$$
\kappa=\tau=\frac{1}{\left(1+t^{2}\right)^{2}}
$$

(cf. Coxeter, exercise 17.8.2.)

Note that a usual name for the twisted cubic (or rational normal curve) is rational helix.

\subsection{Spherical optimal striola}

Our computational models of striola (cf. Methods) belong to a spherical surface $M$ representing the macula. The normal vector $\vec{N}$ along the curve $S$ which represents the polarization of hair cells disposed along $S$ is the normal to the tangent vector $\vec{T}$ which is also tangent to the sphere $M$. The discussion in the preceding section invites to consider as optimal the spherical curves with constant regularized jacobian determinant. We saw two natural choices; the first one, with algebraic coordinates as $\left(\sigma_{1}=s_{1}+s_{2}, \sigma_{2}=s_{1} s_{2}\right)$ or any regular functions of these coordinates, gives a constant product $\kappa \tau^{2}$; the second one with geometric parametrization by $\Sigma$, gives more simply a constant torsion $\tau$.

We suppose that $M$ is part of the sphere in the $3 D$ Euclidian space, centered in 0 of radius $R$. The variable point on the curve $S$ is written now $X(s)$, where $s$ denotes a curvilinear abscissa on $S$. By definition, we have

$$
\dot{X}=\vec{T}, \quad \dot{\vec{T}}=\kappa \vec{p}, \quad \dot{\vec{p}}=-\kappa \vec{T}+\tau \vec{b}
$$

Let us denote $C=1 / \kappa, D=1 / \tau$ the radius of curvature and the radius of torsion respectively. We have

$$
\vec{p}=C \ddot{X}, \quad \dot{\vec{p}}=C \dddot{X}+\dot{C} \ddot{X}
$$

thus

$$
\vec{b}=\frac{1}{\tau} \dot{p}+\frac{\kappa}{\tau} \vec{T}=D C \dddot{X}+D \dot{C} \ddot{X}+D C^{-1} \dot{X}
$$

That gives the following formulas for the tangent, normal and binormal:

$$
\vec{T}=\dot{X}, \quad \vec{p}=C \ddot{X}, \quad \vec{b}=D C \ddot{X}+D \dot{C} \ddot{X}+D C^{-1} \dot{X}
$$

As $X . X$ is constant equal to $R^{2}$, we have $X . \vec{T}=0$, and by derivation of this identity we get $\vec{T} \cdot \vec{T}+\kappa X \cdot \vec{p}=0$. But $\vec{T}$ has a norm equal to 1 , thus we get $X \cdot \vec{p}=-C$, or equivalently $X \cdot \ddot{X}=-1$.

By derivation again, we get $\dot{X} \cdot \ddot{X}+X . \dddot{X}=0$, then $X . \dddot{X}=0$ because $\vec{T}$ and $\vec{p}$ are orthogonal. Then the scalar product $X . \vec{b}$ is equal to $D \dot{C} X . \ddot{X}$ that is $-D \dot{C}$. Thus

$$
X=-C \vec{p}-D \dot{C} \vec{b}
$$


and by equating the norm of $X$ to $R$, this gives the well known intrinsic equation for spherical curves:

$$
C^{2}+D^{2} \dot{C}^{2}=R^{2}
$$

When $D$ is constant, this equation has for only solutions

$$
C(s)=R \cos \left(\frac{s-s_{0}}{D}\right) .
$$

When $D \sqrt{C}$ is constant, equals to $A$, this equation has for solutions

$$
C(s)=R . \operatorname{cnl}^{2}\left(\frac{R^{1 / 2}\left(s-s_{0}\right)}{2 A}\right),
$$

where $c n l$ denotes the lemniscate elliptic function coslemn, that inverses the lemniscate elliptic integral of Bernoulli and Fagnano:

$$
I(u)=\int_{u}^{1} \frac{d t}{\sqrt{1-t^{4}}}
$$

With the Jacobi-Guderman notations we have $\operatorname{cnl}(x)=\operatorname{cn}(x \sqrt{2}, 1 / \sqrt{2})$. (A nice reference for elliptic functions is [23])

The associated functions $\vec{T}, \vec{p}, \vec{b}, X$ can easily be deduced from the solutions of the following two by two linear differential system:

$$
\frac{d Y(s)}{d s}=\frac{i}{2}\left(\begin{array}{cc}
\kappa(s) & \tau(s) \\
-\tau(s) & -\kappa(s)
\end{array}\right) Y(s)
$$

In fact, if $Y(s)$ is a resolvent matrix of this system, and if we introduce the imaginary Pauli matrices

$$
e_{1}=i \sigma_{1}=\left(\begin{array}{cc}
0 & i \\
i & 0
\end{array}\right) \quad e_{2}=i \sigma_{2}=\left(\begin{array}{cc}
0 & 1 \\
-1 & 0
\end{array}\right) \quad e_{3}=i \sigma_{3}=\left(\begin{array}{cc}
i & 0 \\
0 & -i
\end{array}\right)
$$

satisfying

$$
e_{1} e_{2}-e_{2} e_{1}=-2 e_{3}, \quad e_{2} e_{3}-e_{3} e_{2}=-2 e_{1}, \quad e_{3} e_{1}-e_{1} e_{3}=-2 e_{2},
$$

then the matrices

$$
\frac{1}{2} Y e_{1} Y^{-1}, \quad \frac{1}{2} Y e_{2} Y^{-1}, \quad \frac{1}{2} Y e_{3} Y^{-1},
$$

decomposed in the basis $e_{1}, e_{2}, e_{3}$ give respectively the vectorial functions $\vec{T}(s), \vec{p}(s), \vec{b}(s)$. Then the curve $X(s)$ can be deduced by one quadrature.

However there is no known explicit formula for $Y(s)$ when $\kappa$ and $\tau$ correspond to the particular curvature function $C(s)$ we found before. We can only compute their Taylor polynomials. 

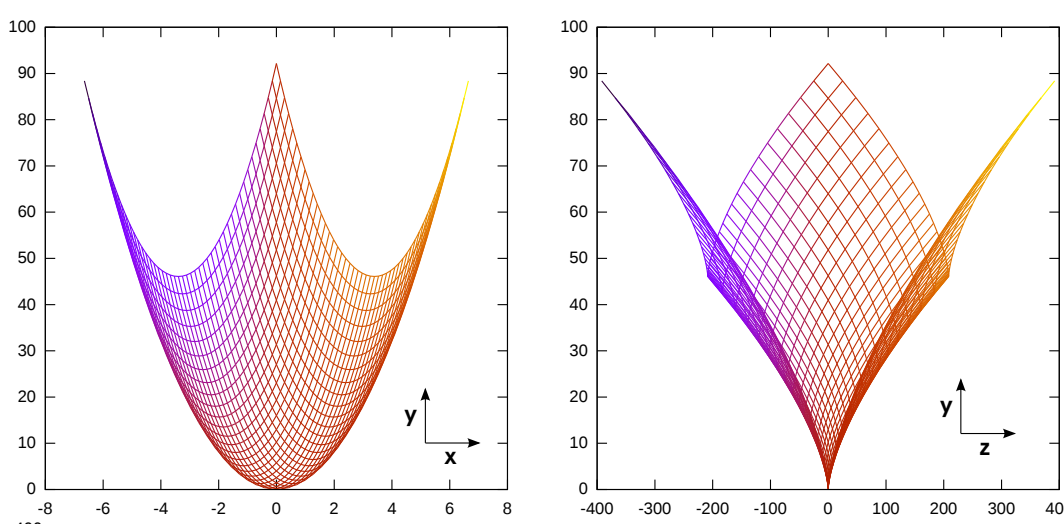

z scale
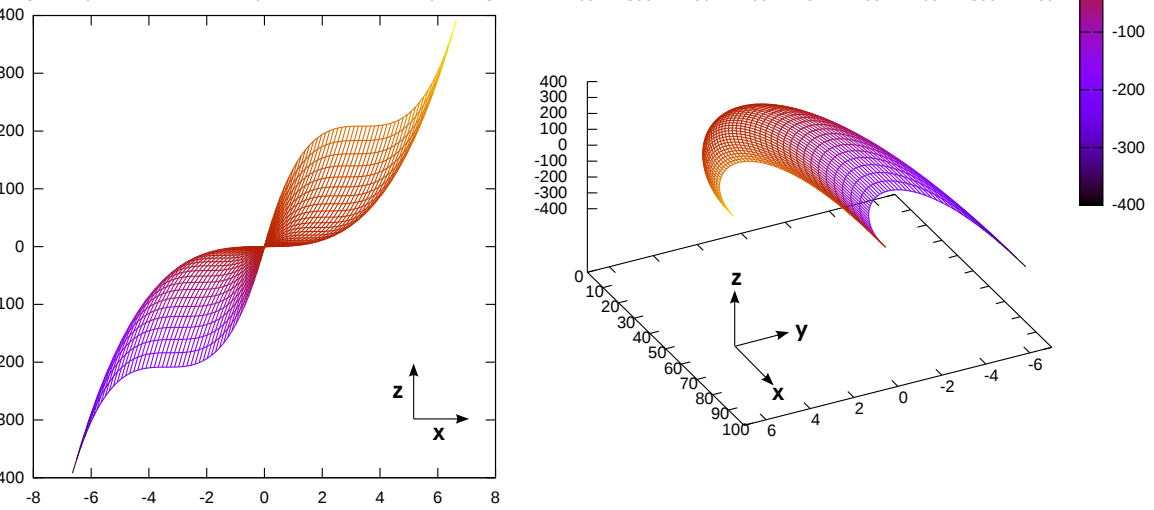

Fig. 15 Representation of the virtual surface $\Sigma$, defined by equations (34-36), whose normals define the perceived directions. It is here computed for the curve $x(t)=t / \sqrt{(2)}, y=$ $t^{2}, z=\sqrt{(2)} / 3 \times t^{3}$, for $t \in[-10 ; 10]$. Panels show the projection of this surface in the $(x, y)$, $(z, y)$ and $(x, z)$ planes, as well as in an arbitrary plane, the color palette represents the same $\mathrm{z}$ value in each of the panels.

\section{References}

1. Berthoz, A., Allain, R., Bennequin, D., David, R., Janvier, P.: Sortir de l'eau. Le passage de la vie aquatique la vie terrestre., chap. Sortir de l'eau et systeme vestibulaire. P.Corvol et J.-L. Elghozi (dir.). Odile Jacob (2011)

2. Rumelhart, D.E. and Hintont, G.E. and Williams, R.J.:Learning representations by back-propagating errors Nature323(6088), 533-536 (1986), London.

3. Boyle, R., Goldberg, J.M., Highstein, S.M.: Inputs from regularly and irregularly discharging vestibular nerve afferents to secondary neurons in squirrel monkey vestibular nuclei. III. Correlation with vestibulospinal and vestibuloocular output pathways. Journal of Neurophysiology 68(2), 471-484 (1992). URL http://jn.physiology.org/content/68/2/471.abstract

4. Brichta, A.M., Goldberg, J.M.: The Papilla Neglecta of Turtles: A Detector of Head Rotations with Unique Sensory Coding Properties. J. Neurosci. 18(11), 4314-4324 (1998). URL http://www.jneurosci.org/cgi/content/abstract/18/11/4314

5. Bronstein, A.M., Gresty, M.A.: Short latency compensatory eye movement responses to transient linear head acceleration: a specific function of the otolith-ocular reflex. Experimental Brain Research 71(2), 406-410 (1988) 
6. Chang, J., Popper, A., Saidel, W.: Heterogeneity of sensory hair cells in a fish ear. J. Comp. Neurol. 4(324), 621-40 (1992)

7. Coxeter, H.: Introduction to Geometry. Wiley and Sons (1969)

8. Curthoys, I.S., Betts, G.A., Burgess, A.M., MacDOUGALL, H.G., Cartwright, A.D., Halmagyi, G.M.: The Planes of the Utricular and Saccular Maculae of the Guinea Pig. Annals of the New York Academy of Sciences 871(1), 27-34 (1999). DOI 10.1111/j.17496632.1999.tb09173.x. URL http://dx.doi.org/10.1111/j.1749-6632.1999.tb09173.x

9. Curthoys, I.S., Black, R.A., Goldberg, J.M., Fernández, C.: New representations of otolithic primary afferent spatial tuning-a re-processing of the Fernández \& Goldberg (1976) data. Acta oto-laryngologica. Supplementum 520 Pt 2, 427-429 (1995). URL http://view.ncbi.nlm.nih.gov/pubmed/8749180

10. Darboux, G.: Leçons sur la Théorie générale des Surfaces et les applications géométriques du Calcul Infinitésimal, vol. 1. Gauthier-Villars (1887)

11. Deliagina, T.G., Beloozerova, I.N., Zelenin, P.V., Orlovsky, G.N.: Spinal and supraspinal postural networks. Brain research reviews $\mathbf{5 7}(1), \quad 212-221 \quad$ (2008). $\quad$ DOI 10.1016/j.brainresrev.2007.06.017. URL http://dx.doi.org/10.1016/j.brainresrev.2007.06.017

12. Desai, S.S., Zeh, C., Lysakowski, A.: Comparative Morphology of Rodent Vestibular Periphery. I. Saccular and Utricular Maculae. Journal of Neurophysiology 93(1), 251266 (2005). DOI 10.1152/jn.00746.2003. URL http://dx.doi.org/10.1152/jn.00746.2003

13. Dickman, J., Angelaki, D., Correia, M.: Response properties of gerbil otolith afferents to small-angle pitch and roll tilts. Brain Research 556, 303-310 (1991)

14. Eatock, R., Songer, J.: Vestibular hair cells and afferents: Two channels for head motion signals. Annu Rev Neurosci 34, 501-534 (2011)

15. Eugène, D., Idoux, E., Beraneck M.and Moore, L., Vidal, P.P.: Intrinsic membrane properties of central vestibular neurons in rodents. Experimental Brain Research pp. 1-14 (2011). DOI 10.1007/s00221-011-2569-3. URL http://dx.doi.org/10.1007/s00221011-2569-3

16. Fernández, C., Goldberg, J.M.: Physiology of peripheral neurons innervating otolith organs of the squirrel monkey. I. Response to static tilts and to longduration centrifugal force. $\mathrm{J}$ Neurophysiol 39(5), 970-984 (1976). URL http://view.ncbi.nlm.nih.gov/pubmed/824412

17. Fernández, C., Goldberg, J.M.: Physiology of peripheral neurons innervating otolith organs of the squirrel monkey. II. Directional selectivity and forceresponse relations. Journal of neurophysiology 39(5), 985-995 (1976). URL http://view.ncbi.nlm.nih.gov/pubmed/824413

18. Fernández, C., Goldberg, J.M.: Physiology of peripheral neurons innervating otolith organs of the squirrel monkey. III. Response dynamics. Journal of neurophysiology 39(5), 996-1008 (1976). URL http://view.ncbi.nlm.nih.gov/pubmed/824414

19. Fernandez, C., Goldberg, J.M., Baird, R.A.: The vestibular nerve of the chinchilla. III. Peripheral innervation patterns in the utricular macula. Journal of Neurophysiology 63(4), 767-780 (1990). URL http://jn.physiology.org/content/63/4/767.abstract

20. Georgopoulos, A., Schwartz, A., Kettner, R.: Neuronal population coding of movement direction. Science 233, 1416-1419 (1986)

21. Goldberg, J.M.: Afferent diversity and the organization of central vestibular pathways. Experimental Brain Research 130(3), 277-297 (2000). DOI 10.1007/s002210050033. URL http://dx.doi.org/10.1007/s002210050033

22. Goldberg, J.M., Desmadryl, G., Baird, R.A., Fernandez, C.: The vestibular nerve of the chinchilla. IV. Discharge properties of utricular afferents. Journal of Neurophysiology 63(4), 781-790 (1990). URL http://jn.physiology.org/content/63/4/781.abstract

23. Greenhill, A.: The applications of elliptic functions. MacMillan and co, New York (1892)

24. Griffith, P., Harris, J.: Principles of Algebraic Geometry. Wiley and Sons (1978)

25. Hess, B.J.: Three-dimensional head angular velocity detection from otolith afferent signals. Biological cybernetics 67(4), 323-333 (1992). URL http://view.ncbi.nlm.nih.gov/pubmed/1515511

26. Highstein, S., Rabbitt, R., Holstein, G., Boyle, R.: Determinants of spatial and temporal coding by semicircular canal afferents. J Neurophysiol 93(5), 2359-2370 (2005) 
27. Jaeger, R.: Modeling the relation between head orientations and otolith responses in humans. Hearing Research 173(1-2), 29-42 (2002). DOI 10.1016/S0378-5955(02)004859. URL http://dx.doi.org/10.1016/S0378-5955(02)00485-9

28. Jaeger, R., Haslwanter, T.: Otolith responses to dynamical stimuli: results of a numerical investigation. Biological Cybernetics 90(3), 165-175 (2004). DOI 10.1007/s00422-0030456-0. URL http://dx.doi.org/10.1007/s00422-003-0456-0

29. Jaeger, R., Kondrachuk, A.V., Haslwanter, T.: The distribution of otolith polarization vectors in mammals: comparison between model predictions and single cell recordings. Hearing research 239(1-2), 12-19 (2008). DOI 10.1016/j.heares.2008.01.004. URL http://dx.doi.org/10.1016/j.heares.2008.01.004

30. Jones, T., Jones, S., Colbert, S.: The adequate stimulus for avian short latency vestibular responses to linear translation. J Vestib. Res. 3(8), 253-72 (1998)

31. Lanford, P.J., Platt, C., Popper, A.N.: Structure and function in the saccule of the goldfish (Carassius auratus): a model of diversity in the non-amniote ear. Hearing research 143(1-2), 1-13 (2000). URL http://view.ncbi.nlm.nih.gov/pubmed/10771179

32. Lange, M., Jones, T.: Short latency electrophysiological responses to pulsed linear acceleration in the mammal. Assoc Res Otolaryngol pp. 343-390 (1990)

33. Leonard, R.: Molecular probes of the vestibular nerve I. Peripheral termination patterns of calretinin, calbindin and peripherin containing fibers. Brain Research 928(1-2), 8-17 (2002). DOI 10.1016/S0006-8993(01)03268-1. URL http://dx.doi.org/10.1016/S00068993(01)03268-1

34. Li, A., Xue, J., Peterson, E.: Architecture of the mouse utricle: macular organization and hair bundle heights. J Neurophysiol 99(2), 718-733 (2007)

35. Li, A., Xue, J., Peterson, E.H.: Architecture of the Mouse Utricle: Macular Organization and Hair Bundle Heights. Journal of Neurophysiology 99(2), 718-733 (2008). DOI 10.1152/jn.00831.2007. URL http://dx.doi.org/10.1152/jn.00831.2007

36. Lin, V., Golub, J., Nguyen, T., Hume, C., Oesterle, E., Stone, J.: Inhibition of notch activity promotes nonmitotic regeneration of hair cells in the adult mouse utricles. The Journal of Neuroscience 31(43), 15,329-15,339 (2011)

37. Lindeman, H.: Studies on the morphology of the sensory regions of the vestibular apparatus. Erg Anat Entw Gesch (42), 1-113 (1969)

38. Lindeman, H.H.: Anatomy of the otolith organs. Advances in oto-rhino-laryngology 20, 405-433 (1973). URL http://view.ncbi.nlm.nih.gov/pubmed/4267996

39. Lorincz, E.N., Hess, B.J.M.: Dynamic Effects on the Subjective Visual Vertical After Roll Rotation. Journal of Neurophysiology 100(2), 657-669 (2008). DOI 10.1152/jn.01248.2007. URL http://dx.doi.org/10.1152/jn.01248.2007

40. Lu, Z.: Morphological polarizations of sensory hair cells in the three otolithic organs of a teleost fish: fluorescent imaging of ciliary bundles. Hearing Research 126(1-2), 47-57 (1998). DOI 10.1016/S0378-5955(98)00149-X. URL http://dx.doi.org/10.1016/S03785955(98)00149-X

41. Lyford-Pike, S., Vogelheim, C., Chu, E., Santina, C.D., Carey, J.: Gentamicin is primarily localized in vestibulat type I hair cells after intratympanic administration. Journal of the Association for Research in Otolaryngology (JARO) (8), 497-508 (2007)

42. Minor, L.B., Goldberg, J.M.: Vestibular-nerve inputs to the vestibulo-ocular reflex: a functional- ablation study in the squirrel monkey. The Journal of Neuroscience 11(6), 1636-1648 (1991). URL http://www.jneurosci.org/content/11/6/1636.abstract

43. Moravec, W., Peterson, E.: Differences between stereocilia numbers on type i and type ii vestibular hair cells. J Neurophysiol 92, 3153-3160 (2004)

44. Naganuma, H., Tokumasu, K., Okamoto, M., Hashimoto, S., Yamashina, S.: Threedimensional analysis of morphological aspects of the human saccular macula. The Annals of otology, rhinology, and laryngology 110(11), 1017-1024 (2001). URL http://view.ncbi.nlm.nih.gov/pubmed/11713911

45. Naganuma, H., Tokumasu K.and Okamoto, M., Hashimoto, S., Yamashina, S.: Three-dimensional analysis of morphological aspects of the human utricular macula. The Annals of otology, rhinology, and laryngology 112(5), 419-424 (2003). URL http://view.ncbi.nlm.nih.gov/pubmed/12784980

46. Nam, J., Cotton, J., Grant, W.: A virtual hair cell, i: evaluation of mechanoelectric transduction parameters. Biophys J 92(6), 1918-1928 (2007) 
47. Nam, J., Cotton, J., Grant, W.: A virtual hair cell, ii: evaluation of mechanoelectric transduction parameters. Biophys J 92(6), 1929-1937 (2007)

48. Peterson, E.: Are there parallel channels in the vestibular nerve? New Physiology Science (13), 194-201 (1988)

49. Platt, C., Jørgensen, J.M., Popper, A.N.: The inner ear of the lungfish Protopterus. J. Comp. Neurol. 471(3), 277-288 (2004). DOI 10.1002/cne.20038. URL http://dx.doi.org/10.1002/cne.20038

50. Rabbitt, R.: Directional coding of three-dimensional movements by the vestibular semicircular canals. Biological Cybernetics 80(6), 417-431 (1999)

51. Ross, M.: Morphological evidence for parallel processing of information in rat macula. Acta Otolaryngol. 106(3-4), 213-218 (1988)

52. Ross, M.: Morphological evidence for local microcircuits in rat vestibular maculae. J. Comp. Neurol. 379(3), 333-346 (1997). DOI 10.1002/(SICI)1096-9861(19970317)379:3\%3C333::AID-CNE2\%3E3.0.CO;2-4. URL http://dx.doi.org/10.1002/(SICI)1096-9861(19970317)379:3\%3C333::AIDCNE2\%3E3.0.CO;2-4

53. Ross, M.: Complex vestibular macular anatomical relationships need a synthetic approach. Acta Otolaryngol Suppl 545 $\left(^{*}\right), 25-28$ (2001)

54. Ross, M.: The evolution of concepts of vestibular peripheral information processing: toward the dynamic, adaptive, parallel processing macular model. Acta oto-laryngologica 123(7), 784-794 (2003). URL http://view.ncbi.nlm.nih.gov/pubmed/14575392

55. Ross, M., Dayhoff, J., Mugler, D.: Toward modeling a dynamic biological neural network. Math Comput Model 13(7), 97-105 (1990)

56. Ross, M., Linton, S., Parnas, B.: Simulation studies of vestibular macular afferentdischarge patterns using a new, quasi-3-d finite volume method. J Comput Neurosci 8(1), 5-18 (2000)

57. Rowe, H., Peterson, E.: Quantitative analysis of stereociliari arrays on vestibular hair cells. Hearing Research 190, 10-24 (2004)

58. Rowe, M., Peterson, E.: Autocorrelation analysis of hair bundle structure in the utricle. J Neurophysiol 96(5), 2653-2669 (2006)

59. Sato, H., Sando, I., Takahashi, H.: Computer-aided three-dimensional measurement of the human vestibular apparatus. Otolaryngology-head and neck surgery : official journal of American Academy of Otolaryngology-Head and Neck Surgery 107(3), 405409 (1992). URL http://view.ncbi.nlm.nih.gov/pubmed/1408226

60. Si, X., Angelaki, D., Dickman, J.: Response properties of pigeon otolith afferents to linear acceleration. Exp Brain Res 117, 242-250 (1997)

61. Si, X., Zakir, M.M., Dickman, J.D.: Afferent Innervation of the Utricular Macula in Pigeons. Journal of Neurophysiology 89(3), 1660-1677 (2003). DOI 10.1152/jn.00690.2002. URL http://dx.doi.org/10.1152/jn.00690.2002

62. Simmons, D.D., Tong, B., Schrader, A.D., Hornak, A.J.: Oncomodulin identifies different hair cell types in the mammalian inner ear. J. Comp. Neurol. 518(18), 3785-3802 (2010). DOI 10.1002/cne.22424. URL http://dx.doi.org/10.1002/cne.22424

63. Smith, C., Goldberg, J.: A stochastic afterhyperpolarization model of repetitive activity in vestibular afferents. Biological Cybernetics 54(1), 41-51 (1986)

64. Spoedlin, H.: The Vestibular System and Its Deseases, chap. The ultrastructure of the vestibular sense organ. Wolfson, R.J. (Philadelphia: University of Pennsylvania Press) (1966)

65. Spoon, C., Moravec, W., Rowe, M., Grant, J., Peterson, E.: Steady-state stiffness of utricular hair cells depends on macular location and hair bundle structure. J Neurophysiol 106(6), 2950-63 (2011)

66. Straka, H., Beraneck, M., Rohregger, M., Moore, L.E., Vidal, P.P., Vibert, N.: SecondOrder Vestibular Neurons Form Separate Populations With Different Membrane and Discharge Properties. Journal of Neurophysiology 92(2), 845-861 (2004). DOI 10.1152/jn.00107.2004. URL http://dx.doi.org/10.1152/jn.00107.2004

67. Takagi, A., Sando, I.: Computer-aided three-dimensional reconstruction and measurement of the vestibular end-organs. Otolaryngology-head and neck surgery : official journal of American Academy of Otolaryngology-Head and Neck Surgery 98(3), 195202 (1988). URL http://view.ncbi.nlm.nih.gov/pubmed/2895448 
68. Tomko, D., Peterka, R., Schor, R., O'Leary, D.: Response dynamics of horizontal canal afferents in barbiturate-anesthetized cats. J Neurophysiol 45(3), 376-396 (1981)

69. Tribukait, A., Rosenhall, U.: Directional sensitivity of the human macula utriculi based on morphological characteristics. Audiology \& neuro-otology 6(2), 98-107 (2001). URL http://view.ncbi.nlm.nih.gov/pubmed/11385183

70. Tribukait, A., Rosenhall, U., Osterdahl, B.: Morphological characteristics of the human macula sacculi. Audiology \& neuro-otology 10(2), 90-96 (2005). DOI 10.1159/000083364. URL http://dx.doi.org/10.1159/000083364

71. Vilares, I., Kording, K.: Bayesian models: the structure of the world, uncertainty, behavior, and the brain. Annals of New-York Adademy of Sciences 1224, 22-39 (2011)

72. Watanuki, K., Kawamoto, K., Katagiri, S.: Structure of the otolithic layers on the maculae sacculi and utriculi in the guinea pig. Equilibrium Res Suppl 2 pp. 41-48 (1971)

73. Xue, J., Peterson, E.: Hair bundle heights on the utricle: Differences between macular locations and hair cell types. J Neurophysiol 95, 176-186 (2006)

74. Zakir, M., Huss, D., Dickman, J.D.: Afferent Innervation Patterns of the Saccule in Pigeons. Journal of Neurophysiology 89(1), 534-550 (2003). DOI 10.1152/jn.00817.2001. URL http://dx.doi.org/10.1152/jn.00817.2001 